\title{
Dynamical Modelling of Bone Formation and Resorption under Impulsive Estrogen Supplement: Effects of Parathyroid Hormone and Prolactin
}

\author{
Supassorn Aekthong ${ }^{1}$ and Chontita Rattanakul $\mathbb{D}^{1,2}$ \\ ${ }^{1}$ Department of Mathematics, Faculty of Science, Mahidol University, Bangkok 10400, Thailand \\ ${ }^{2}$ Centre of Excellence in Mathematics, Commission on Higher Education, Bangkok 10400, Thailand \\ Correspondence should be addressed to Chontita Rattanakul; chontita.rat@mahidol.ac.th
}

Received 21 September 2021; Accepted 1 December 2021; Published 15 December 2021

Academic Editor: Hernando Quevedo

Copyright (c) 2021 Supassorn Aekthong and Chontita Rattanakul. This is an open access article distributed under the Creative Commons Attribution License, which permits unrestricted use, distribution, and reproduction in any medium, provided the original work is properly cited.

\begin{abstract}
Osteoporosis, a bone metabolic disease, is one of the major diseases occurring in aging population especially in postmenopausal women. A system of impulsive differential equations is developed in this paper in order to investigate the effects of parathyroid hormone and prolactin on bone-forming cells, namely, osteoblasts, and bone-resorbing cells, namely, osteoclasts, under the impulsive estrogen supplement. The theoretical analysis of the developed model is carried out so that we obtain the conditions on the system parameters in which the stability and permanence of the model can occur. Computer simulations are also provided to illustrate the theoretical predictions.
\end{abstract}

\section{Introduction}

Osteoporosis is a disease which shows no symptoms until there is a bone fracture incidence [1]. It is characterized by an impairment of bone mass, measured by a Bone Mineral Density (BMD) test as the T-score is less than or equal to 2.5, and microarchitectural deterioration of bone tissue [2-5]. This disease causes a major public health issue for all races and both sexes [1]. Since BMD decreases as the population ages, osteoporosis occurs prevalently in menopause woman and elderly men between 75 and 80 years of age [6]. More than 200 million people worldwide were approximated to be osteoporotic patients with hip fractures [7]. In Europe and the United States, a report revealed that $30 \%$ of women are diagnosed with osteoporosis [8]. It was reported in Thailand that $19.8 \%$ of postmenopausal women are prevalently osteoporotic [9]. Furthermore, approximately $40 \%$ of women who experience menopause and $30 \%$ of men might have an osteoporosis-related fracture in the remaining lifetime [10].

Bone is a living tissue [11]. There is a process in its place to resorb old bone and form new bone to maintain its strength and health, called bone-remodeling process, and such a process occurs throughout a person's life [10, 12, 13]. In osteoporosis patients, the loss of bone mass occurs because the removal of older bone is more than the replacement of new bone which is an imbalance between bone resorption and bone formation [10]. There are two families of cells mainly involved in the process of bone remodeling, namely, osteoclastic cells, breaking down the bone cells, and osteoblastic cells, forming the bone tissue [12, 13]. Boneremodeling process is composed of four phases sequentially, which are activation, resorption, reversal, and formation [12]. In this process, osteoblast lineage cells play a crucial role in the activation stage by taking action on blood cell precursors or hematopoietic cells to establish osteoclastic resorption of bones. Under a layer of lining cells, the removal of bone is done by osteoclasts in the resorption step. The mineral here is dissolved, and the bone matrix is broken down. In the reversal phase, the surface of the resorbed bone creates a thin layer of protein to make it ready for the following stage. Finally, in the formation step, osteoblasts acting in successive waves start to form up new bones by 
laying down multiple layers of matrix in an orderly arrangement. Then, some of these osteoblasts stay inside the bone and become osteocytes, remaining in contact to adjacent osteocytes and osteoblasts on the bone surface. This process is successfully finished in 3-4 months, while the first three stages take 2 to 3 weeks to finish [12]. There are many factors involved in bone-remodeling process such as parathyroid hormone $(\mathrm{PTH})$, prolactin (PRL), calcitonin, and estrogen.

PTH plays an important role in the bone-remodeling process [14]. It is released from the parathyroid gland, and both stimulating and inhibiting effects have been reported on the development of osteoblasts from its progenitors [14-18]. On the one hand, PTH indirectly enhances the development of osteoclasts from its progenitors by operating through osteoblasts since osteoclasts lack PTH receptors while preosteoblast precursors and preosteoblasts possess them [14-18]. On the other hand, PRL is a polypeptide hormone synthesized in and secreted from specialized cells of the anterior pituitary gland, the lactotrophs [19]. According to [20], PRL receptors have been reported on osteoblasts, and hence, PRL then has effects on boneremodeling process as well. PRL enhances bone resorption by increasing receptor activator of NF-ligand (RANKL) and decreasing osteoprotegerin (OPG) expressions by osteoblastic cells [21].

Bone mass reduces significantly due to the declining secretion of estrogen during and after the menopause years in women [22]. Estrogen deficiency in postmenopausal women leads to accelerating resorption of bone by osteoclasts, and osteoporosis will then occur [23]. The direct and indirect studies indicated that estrogen also plays a key role on skeletal health in men [24]. Several hormones play an important role in the process of bone remodeling and also in the treatments of osteoporotic patients. Estrogen replacement therapy has a beneficial effect on postmenopausal women in preventing the loss of bone as shown in clinical studies $[25,26]$. In a long-term study of following up postmenopausal women who take estrogen replacement therapy, in vivo study results demonstrated that replacement estrogen therapy can prevent or slow down osteoporosis in postmenopausal patients compared to an unsupplemented control group [27]. A recent research using cancellous rats reported that decreased estrogen levels led to bone changes which affect flow of interstitial around osteocytes [28]. Many studies have shown that estrogen impacts positively in improving bone mineral density, and taking lower doses of estrogen is shown to be effective and cause fewer side effects. At the cellular level, estrogen increases the level of OPG which is able to bind with RANKL to block the differentiation, the activity, and the survival of osteoclasts.

Since bone is an alive and dynamic tissue [11], many researchers have been trying to describe the process of bone remodeling inside our bodies using several types of mathematical models. Chudtong et al. [29] introduced an impulsive system to describe bone-remodeling process involving PTH supplements by extending Rattanakul et al.'s model in [30]. Rattanakul et al. [31] constructed a mathematical model as a system of nonlinear differential equations to investigate bone formation and resorption process based on the effect of calcitonin. Motivated by [31], Panitsupakamon and Rattanakul [32] modified the model proposed in [31] by incorporating the time delay observed in the bone-remodeling process. The influence of estrogen supplement is also considered by adding a term to the dynamics of the active osteoclast population in the modified model. Chaiya and Rattanakul [33] proposed an impulsive mathematical model of bone-remodeling process incorporating the effects of prolactin and the impulsive control strategies of parathyroid hormone supplement on osteoblasts and osteoclasts. The dosage of parathyroid hormone can be added appropriately to the system to ensure the suitable levels of active osteoblasts and active osteoclasts. They also proposed another model accounting for the effects of PTH and calcitonin on bone-remodeling process together with the effects of impulsive treatments of estrogen in [34]. However, a mathematical model of bone resorption and bone formation consisting of osteoblastic cells, osteoclastic cells, parathyroid hormone, and prolactin with the effect of impulsive treatment of estrogen has never been established.

\section{Model Development}

In this section, we propose the following impulsive differential equation model to investigate the dynamics of boneforming cells and bone-resorbing cells based on the effects of PTH, PRL, and estrogen supplements:

$$
\left.\begin{array}{l}
\frac{\mathrm{d} x}{\mathrm{~d} t}=\frac{a_{1}}{k_{1}+z}+a_{2} y-b_{1} x, \\
\frac{\mathrm{d} y}{\mathrm{~d} t}=\frac{a_{3}}{k_{2}+z}+a_{4} x-b_{2} y, \\
\frac{\mathrm{d} z}{\mathrm{~d} t}=\left[\frac{a_{5} x}{\left(k_{3}+x^{2}\right)}+\frac{a_{6} y}{\left(k_{4}+y^{2}\right)}\right] z w-b_{3} z, \\
\frac{\mathrm{d} w}{\mathrm{~d} t}=a_{7} x-\frac{a_{8} x w}{k_{5}+x}-\frac{a_{9} y w}{k_{6}+y}-b_{4} w,
\end{array}\right\} t \neq n T,
$$

with

$$
\left.\begin{array}{l}
\Delta z(t)=-\rho z(t), \\
\Delta w(t)=\mu,
\end{array}\right\} t=n T,
$$

where $\Delta z(t)=z\left(t^{+}\right)-z(t)$ and $\Delta w(t)=w\left(t^{+}\right)-w(t)$. In what follows, $x(t), y(t), z(t)$, and $w(t)$ account for the level of PTH in blood at time $t$, the level of PRL in blood at time $t$, the number of active osteoclasts at time $t$, and the number of active osteoblasts at time $t$, respectively. $T$ accounts for the period between each impulsive estrogen treatment, $n \in Z_{+}=$ $\{1,2,3, \ldots\}, \rho$ accounts for the inhibiting effect of estrogen treatment on osteoclasts, $0<\rho<1$, and $\mu$ accounts for the stimulating effect on osteoblasts of estrogen treatment, $\mu>0$. In addition, all parameters in (1) and (2) are assumed to be positive. 
PTH secretion from the parathyroid gland is controlled by the calcium level in blood. Once the number of active osteoclasts increases, blood calcium levels then increase due to the increase in bone resorption resulting in the decrease of PTH secretion in order to maintain calcium level within the normal range $[35,36]$. Hence, we then assumed that the rate of change of the concentration of PTH in blood at time $t$ denoted by $\mathrm{d} x / \mathrm{d} t$ in equation (1) varies inversely with the number of active osteoclasts as shown in the first term on the right-hand side of the first equation in equation (1). According to [37], an increase in the level of plasma PRL also enhances the release of PTH from the parathyroid gland and hence the second term on the right-hand side denotes the stimulating effect of PRL on PTH. The last term stands for the removal rate of PTH from the system.

However, PRL stimulates osteoclastic differentiation by decreasing OPG and increasing RANKL expressions by osteoblastic cells [21]. RANKL then binds to RANK, its receptor on preosteoclast, and stimulates the differentiation of osteoclasts. To balance the bone-remodeling cycle, when the number of osteoclasts increases, the secretion of PRL is then decreased. In the second equation of (1), the rate of change of the level of PRL in blood denoted by $\mathrm{d} y / \mathrm{d} t$ is then assumed to vary inversely with the number of active osteoclasts as shown in the first term on the right-hand side. On the other hand, the increase in the number of osteoclasts leads to the increase in the calcium level in blood and then the secretion of PTH from the parathyroid gland, as well as the secretion of PRL from the anterior pituitary gland, which also enhances the secretion of PTH will be decreased in order to maintain the calcium level in blood within the normal range $[35,36]$. Then, the second term on the righthand side stands for the stimulating effect of PTH on the release of PRL from the anterior pituitary gland. The last term stands for the removal rate of PRL from the system.

Osteoclasts lack PTH and PRL receptors, whereas osteoblasts possess them. Therefore, the effects of PTH and PRL on the differentiation of osteoclasts are both indirect effects. However, it has been reported in [17] that the differentiation of active osteoclasts also requires the production of osteoclast differentiation factor (ODF) and its receptor on osteoclasts as well and hence the differentiation of active osteoclasts requires the presence of both osteoblastic and osteoclastic cells. Even though PTH has the stimulating effects on the differentiation of active osteoclasts as reported in $[18,38]$, it has also been observed clinically in [17] that PTH inhibits the differentiation of active osteoclasts when the level of PTH increases further as well. On the other hand,
PRL enhances bone resorption by decreasing OPG and increasing RANKL expressions by osteoblastic cells [21]. RANKL then binds to RANK, its receptor on preosteoclast, and stimulates the differentiation of osteoclasts [21]. Thus, the rate of change of the number of active osteoclasts denoted by $\mathrm{d} z / \mathrm{d} t$ is then assumed to depend on the stimulating and inhibiting effects of PTH and PRL as presented on the first term on the right-hand side of the third equation in (1). The last term stands for the removal rate of active osteoclasts.

Osteoblasts possess both PTH receptors and PRL receptors, and hence, the effects of PTH and PRL on the differentiation of osteoblasts are both direct effects. PTH prevents a suicidal process called apoptosis of osteoblasts $[39,40]$. In addition, both stimulating and inhibiting effects of PTH on osteoblastic differentiation process have been clinically observed depending on the differentiation stages [14]. As for the stimulating effect of PTH on the differentiation of osteoblasts, we assumed that the rate of change of the number of active osteoblasts denoted by $\mathrm{d} w / \mathrm{d} t$ varies directly with the level of PTH as presented on the first term on the right-hand side of the fourth equation of (1), whereas the inhibiting effect of PTH on the differentiation of osteoblasts is presented on the second term on the right-hand side. On the other hand, it has been reported in [41] that the osteoblast number (DNA content) is declined significantly due to PRL, resulting in a decrease in osteoblast proliferation, and hence, the third term on the right-hand side is assumed to represent the inhibiting effect of PRL on osteoblasts. The last term represents the removal rate of active osteoblasts.

The first equation of (2) accounts for the inhibitory effect of impulsive treatment with estrogen supplement on the number of active osteoclasts. The second equation accounts for the simulative effect of impulsive treatment with estrogen supplement on the number of active osteoblasts.

Several clinical observations indicate that the dynamics of PTH and PRL are so much faster than the dynamics of osteoclasts and osteoblasts $[35,36,42,43]$. In what follows, PTH and PRL are then assumed to equilibrate rapidly to their equilibrium for which $\mathrm{d} x / \mathrm{d} t=0$ and $\mathrm{d} y / \mathrm{d} t=0$, respectively. That is,

$$
x=\frac{a_{1} b_{2}}{A\left(k_{1}+z\right)}+\frac{a_{2} a_{3}}{A\left(k_{2}+z\right)} \equiv f_{1}(z)
$$

and

$$
y=\frac{a_{3}}{b_{2}\left(k_{2}+z\right)}+\frac{a_{4} f_{1}(z)}{b_{2}}=\frac{a_{3}}{b_{2}\left(k_{2}+z\right)}+\frac{a_{1} a_{4}}{A\left(0 k_{1}+z\right)}+\frac{a_{2} a_{3} a_{4}}{A b_{2}\left(k_{2}+z\right)} \equiv f_{2}(z),
$$

where $A=b_{1} b_{2}-a_{2} a_{4}$ with $b_{1} b_{2}>a_{2} a_{4}$.

Thus, the reduced system of (1) and (2) can be obtained as follows: 


$$
\left.\begin{array}{l}
\frac{\mathrm{d} z}{\mathrm{~d} t}=\left[\frac{a_{5} f_{1}(z)}{\left(k_{3}+f_{1}^{2}(z)\right)}+\frac{a_{6} f_{2}(z)}{\left(k_{4}+f_{2}^{2}(z)\right)}\right] z w-b_{3} z, \\
\frac{\mathrm{d} w}{\mathrm{~d} t}=a_{7} f_{1}(z)-\frac{a_{8} f_{1}(z) w}{k_{5}+f_{1}(z)}-\frac{a_{9} f_{2}(z) w}{k_{6}+f_{2}(z)}-b_{4} w,
\end{array}\right\} t \neq n T,
$$

with

$$
\left.\begin{array}{l}
\Delta z(t)=-\rho z(t), \\
\Delta w(t)=\mu,
\end{array}\right\} t=n T .
$$

\section{Stability Analysis}

Now, we let

$$
U: R_{+} \times R_{+}^{2} \longrightarrow R_{+} \text {, }
$$

where $R_{+}=[0, \infty), R_{+}^{2}=\left\{S \in R^{2}: S=(z, w), z, w \in R_{+}\right\}$. Let us denote the map defined by the right-hand side of (5) and (6) by $F=\left(F_{1}, F_{2}\right)$.

Definition 1. Let $U$ be a continuous function in $(n T,(n+$ 1)T] $\times R_{+}^{2} \longrightarrow R_{+}$. If

$$
\lim _{(t, Y) \longrightarrow\left(n T^{+}, S\right)} U(t, Y)=U\left(n T^{+}, S\right)
$$

exists and is finite for each $S \in R_{+}^{2}, n \in Z_{+}$, and $V$ being locally Lipschitzian in $S$, then the upper right derivative of $U(t, S)$ with respect to (5) and (6) is defined as

$$
D^{+} U(t, S)=\lim _{h \longrightarrow 0^{+}} \sup \frac{1}{h}[U(t+h, S+h F(t, S))-U(t, S)],
$$

where $F=\left(F_{1}, F_{2}\right)$.

The solution $S(t)=(z(t), w(t))$ to systems (5) and (6) is assumed to be a piecewise continuous function which means that $S(t)$ is continuous on $(n T,(n+1) T]$ for $n \in Z_{+}$and $\lim _{t \longrightarrow n T^{+}} S(t)=S\left(n T^{+}\right)$exists. According to the smoothness properties of $F$ [44], the solution to systems (5) and (6) exists and is unique. For $t \neq n T$, we can see that $\mathrm{d} z / \mathrm{d} t=0$ if $z(t)=$ 0 and $\mathrm{d} w / \mathrm{d} t>0$ if $w(t)=0$. Furthermore, for $t=n T, Z\left(n T^{+}\right)=(1-\rho) z(n T)$, where $0<\rho<1 \quad$ and $w\left(n T^{+}\right)=w(n T)+$, where $\mu>0$.

Lemma 1. For all $t \geq 0$, the solution of systems (5) and (6), $S(t)=(z(t), w(t))$, is nonnegative provided that $S\left(0^{+}\right) \geq 0$. In addition, $S(t)$ is positive for all $t \geq 0$ provided that $S\left(0^{+}\right)>0$.

Lemma 2. Suppose that $(z(t), w(t))$ is a solution to (5) and (6) and

$$
b_{4}>\frac{a_{5} M_{3}}{k_{3}}+\frac{a_{6} M_{4}}{k_{4}} .
$$

Then, for sufficiently large $t$, there exists a positive constant $M$ such that $z(t) \leq M$ and $w(t) \leq M$.

Proof. Let $\quad u(t)=z(t)+w(t), \quad \sup f_{1}(z)=M_{1}, \sup z f_{1}$ $(z)=(1 / A)\left(a_{1} b_{2}+a_{2} a_{3}\right)=M_{3}, \quad \sup z f_{2}(z)=\left(a_{3} / b_{2}\right)$ $+\left(a_{1} a_{4} / A\right)+\left(a_{2} a_{3} a_{4} / A b_{2}\right)=M_{4}, \quad$ and $\quad c=\min \left\{b_{3}, b_{4}\right.$ $\left.-\left(a_{5} M_{3} / k_{3}\right)-\left(a_{6} M_{4} / k_{4}\right)\right\}$.

For $t \neq n T$, it follows that

$$
\begin{aligned}
D^{+} u+c u= & \frac{\mathrm{d} z}{\mathrm{~d} t}+\frac{\mathrm{d} w}{\mathrm{~d} t}+c z+c w \\
= & {\left[\frac{a_{5} f_{1}(z)}{\left(k_{3}+f_{1}^{2}(z)\right)}+\frac{a_{6} f_{2}(z)}{\left(k_{4}+f_{2}^{2}(z)\right)}\right] z w-b_{3} z+a_{7} f_{1}(z) } \\
& -\frac{a_{8} f_{1}(z) w}{k_{5}+f_{1}(z)}-\frac{a_{9} f_{2}(z) w}{k_{6}+f_{2}(z)}-b_{4} w+c z+c w \\
\leq & {\left[\frac{a_{5} z f_{1}(z)}{k_{3}+f_{1}^{2}(z)}+\frac{a_{6} z f_{2}(z)}{k_{3}+f_{1}^{2}(z)}\right] w+\left(c-b_{3}\right) z+\left(c-b_{4}\right) w+a_{7} M_{1} } \\
\leq & {\left[\frac{a_{5} M_{3}}{k_{3}}+\frac{a_{6} M_{4}}{k_{4}}+c-b_{4}\right] w+\left(c-b_{3}\right) z+a_{7} M_{1} } \\
\leq & a_{7} M_{1} \equiv M_{0} .
\end{aligned}
$$

Thus, $D^{+} u \leq-c u+M_{0}$. For $t=n T$, we can see that 


$$
\begin{aligned}
u\left(n T^{+}\right) & =z\left(n T^{+}\right)+w\left(n T^{+}\right) \\
& =(1-\rho) z(n T)+\mu+w(n T) \\
& =z(n T)+w(n T)+\mu-\rho z(n T) \\
& \leq u(n T)+\mu .
\end{aligned}
$$

$$
\begin{aligned}
u(t) & \leq u(0) e_{0}^{t}-c d s+\int_{0}^{t} e \int_{s}^{t}-c d \tau M_{0} d s+\sum_{0<t_{n}<t} e \int_{t_{n}}^{t}-c d \tau \\
& =u(0) e^{-c t}+M_{0} \int_{0}^{t} e^{-c(t-s)} d s+\sum_{0<t_{n}<t} \mu e^{-c\left(t-t_{n}\right)} \\
& =u(0) e^{-c t}+M_{0}\left(\frac{1}{c}-\frac{e^{-c t}}{c}\right)+\mu\left(\frac{e^{-c(t-T)}-e^{-c\left(t-t_{n+1}\right)}}{1-e^{c T}}\right) \\
& <\frac{M_{0}}{c}+\mu \frac{e^{-c(t-T)}}{1-e^{c T}}+\mu \frac{e^{c T}}{e^{c T}-1} \leq \frac{M_{0}}{c}+\mu \frac{e^{c T}}{e^{c T}-1} \equiv M .
\end{aligned}
$$

Therefore, $u(t) \leq M$ as $t \longrightarrow \infty$ and $u(t)$ is uniformly ultimately bounded, which means that when $t$ is large enough with $z(t) \leq M$ and $w(t) \leq M$ for some positive constant $M$.

Let us consider the following reduced impulsive system of (5) and (6) when osteoclasts are absent $(z=0)$ :

$$
\begin{aligned}
\frac{\mathrm{d} w}{\mathrm{~d} t} & =B-C w, \quad t \neq n T, \\
w\left(n T^{+}\right) & =w(n T)+\mu, \quad t=n T, \\
w\left(0^{+}\right) & =w_{0},
\end{aligned}
$$

where $B=a_{7} f_{1}(0), C=\left(a_{8} f_{1}(0) / k_{5}+f_{1}(0)\right)+\left(a_{9} f_{2}(0)\right.$ $\left./ k_{6}+f_{2}(0)\right)+b_{4}, f_{1}(0)=\left(a_{1} b_{2} / A k_{1}\right)+\left(a_{2} a_{3} / A k_{2}\right)$ and $f_{2}(0)=a_{3} / b_{2} k_{2}+a_{4} f_{1}(0) / b_{2}$.

It is obvious that $B$ and $C$ are positive provided that $b_{1} b_{2}>a_{2} a_{4}$. By solving system (14), we obtain its periodic solution as follows:

$$
\widetilde{w}(t)=\frac{\mu e^{-C(t-n T)}}{1-e^{-C T}}+\frac{B}{C}, \quad t \in(n T,(n+1) T],
$$

such that $\tilde{w}\left(0^{+}\right)=\mu / 1-e^{-C T}+B / C>0$.

Therefore, the positive solution to system (14) can be written as

$$
w(t)=\left(w_{0}-\frac{B}{C}-\frac{\mu}{1-e^{-C T}}\right) e^{-C t}+\widetilde{w}(t), \quad t \in(n T,(n+1) T] .
$$

Lemma 3. System (14) has a positive periodic solution $\widetilde{w}(t)$. In addition, the solution $w(t)$ of (14) converges to $\widetilde{w}(t)$ as $t \longrightarrow \infty$.
Hence, the positive periodic solution to systems (5) and (6) in the absence of osteoclasts is

$$
(0, \widetilde{w}(t))=\left(0, \frac{\mu e^{-C(t-n T)}}{1-e^{-C T}}+\frac{B}{C}\right),
$$

for $t \in(n T,(n+1) T] \quad$ with $\widetilde{w}\left(n T^{+}\right)=\widetilde{w}\left(0^{+}\right)=(\mu / 1$ $\left.-e^{-C T}\right)+(B / C), n \in Z_{+}$.

Theorem 1. The solution $(0, \widetilde{w}(t))$ of systems (5) and (6) is locally asymptotically stable if

$$
\begin{gathered}
0<T<T_{\text {max }}, \\
b_{3}<\frac{D B}{C}, \\
\frac{D \mu}{C}<\ln \left(\frac{1}{1-\rho}\right),
\end{gathered}
$$

where $D=\left(a_{5} f_{1}(0) / k_{3}+f_{1}^{2}(0)\right)+\left(a_{6} f_{2}(0) / k_{4}+f_{2}^{2}(0)\right)$ and $T_{\max } \equiv\left(1 /\left(D B / C-b_{3}\right)\right)[\ln (1 / 1-\rho)-D \mu / C]$.

Proof. The local stability of the solution $(0, \widetilde{w}(t))$ may be determined by considering the behavior of small amplitude perturbations of the solution.

Here, we let

$$
\begin{aligned}
z(t) & =u_{1}(t), \\
w(t) & =\widetilde{w}(t)+u_{2}(t) .
\end{aligned}
$$

It follows that

$$
\left(\begin{array}{l}
u_{1}(t) \\
u_{2}(t)
\end{array}\right)=\Phi(t)\left(\begin{array}{l}
u_{1}(0) \\
u_{2}(0)
\end{array}\right), \quad 0<t<T,
$$


where $\Phi(t)$ is the fundamental solution matrix, in which

$$
\frac{d \Phi(t)}{\mathrm{d} t}=\left(\begin{array}{cc}
D \widetilde{w}(t)-b_{3} & 0 \\
* & -C
\end{array}\right) \Phi(t),
$$

and $\Phi(0)$ is the identity matrix $I$.

Thus, we obtain the fundamental solution matrix as follows:

$$
\Phi(t)=\left(\begin{array}{cc}
e^{\int_{0}^{t}\left(D \tilde{w}(s)-b_{3}\right) \mathrm{d} s} & 0 \\
* * & e^{\int_{0}^{t}(-C) \mathrm{d} s}
\end{array}\right) .
$$

Note that the terms $*$ and $* *$ are not required in further analysis and their exact expressions are not necessary.

Linearization of (6) provides

$$
\left(\begin{array}{l}
u_{1}\left(n T^{+}\right) \\
u_{2}\left(n T^{+}\right)
\end{array}\right)=\left(\begin{array}{cc}
1-\rho & 0 \\
0 & 1
\end{array}\right)\left(\begin{array}{l}
u_{1}(n T) \\
u_{2}(n T)
\end{array}\right) .
$$

Next, the Floquet theory is then applied to guarantee the local stability of the solution $(0, \widetilde{w}(t))$.

Let us consider

$$
M=\left(\begin{array}{cc}
1-\rho & 0 \\
0 & 1
\end{array}\right) \Phi(T) .
$$

The solution $(0, \widetilde{w}(t))$ is locally asymptotically stable if the magnitudes of $\lambda_{1}$ and $\lambda_{2}$ and the eigenvalue of $M$ are both less than 1 .

We can see that

$$
\begin{aligned}
& \lambda_{1}=(1-\rho) e^{\int_{0}^{T}\left(D \tilde{w}(s)-b_{3}\right) \mathrm{d} s}=(1-\rho) e^{(D \mu / C)+(D B T / C)-b_{3} T}, \\
& \lambda_{2}=e^{\int_{0}^{T}(-C) \mathrm{d} s}=e^{-C T} .
\end{aligned}
$$

Since $0<\rho<1$ and $C>0$, it follows that $\left|\lambda_{1}\right|<1$ provided that (18)-(20) are satisfied. Also, it is obvious that $\left|\lambda_{2}\right|<1$, and hence, the proof is complete.

\section{Permanence of the System}

Definition 2. Systems (5) and (6) are said to be permanent if there exist positive constants $m, M$, and $t_{0}$ such that for all solutions $(z(t), w(t))$ with positive initial values $z\left(0^{+}\right)$and $w\left(0^{+}\right)$the following conditions hold for all $t>t_{0}>0$ :

$$
\begin{aligned}
& m \leq z(t) \leq M, \\
& m \leq w(t) \leq M .
\end{aligned}
$$

Theorem 2. Suppose that

$$
T>T^{*}
$$

where $T^{*} \equiv\left(1 /(D B / C)-b_{3}\right) \ln (1 / 1-\rho)$. Systems (5) and (6) are permanent provided that (10), (19), and (30) hold.

Proof. Let $S(t)=(z(t), w(t))$ be any solution of (5) and (6) with $z\left(0^{+}\right)>0$ and $w\left(0^{+}\right)>0$. Suppose that (10) holds; it follows that when $t$ is large enough, $z(t) \leq M$ and $w(t) \leq M$ for some $M>0$ according to Lemma 2.

Consider the second equation of (5); we can see that

$$
\begin{gathered}
\frac{\mathrm{d} w}{\mathrm{~d} t} \geq-\frac{a_{8} f_{1}(z) w}{k_{5}+f_{1}(z)}-\frac{a_{9} f_{2}(z) w}{k_{6}+f_{2}(z)} \\
-b_{4} w \geq-C w, \quad t \neq n T, \\
w\left(n T^{+}\right)=w(n T)+\mu, \quad t=n T .
\end{gathered}
$$

It follows that for sufficiently large $t$, there exists $\varepsilon>0$ such that

$$
w(t)>-\frac{B}{C}+\widetilde{w}(t)-\varepsilon .
$$

Therefore, we obtain

$$
w(t)>\frac{\mu e^{-C T}}{1-e^{-C T}}-\varepsilon \equiv m_{1},
$$

when $t$ is large enough.

Next, we will show that there is a positive constant $m_{2}$ for which $z(t)>m_{2}$. Firstly, for some $m_{3}>0$, we let

$$
\begin{aligned}
& \widehat{M}_{1}=\frac{a_{5} f_{1}\left(m_{3}\right)}{\left(k_{3}+f_{1}^{2}\left(m_{3}\right)\right)}+\frac{a_{6} f_{2}\left(m_{3}\right)}{\left(k_{4}+f_{2}^{2}\left(m_{3}\right)\right)}, \\
& \widehat{M}_{2}=\frac{a_{1} a_{7} b_{2} k_{2}+a_{2} a_{3} a_{7} k_{1}}{C A\left(k_{1}+m_{3}\right)\left(k_{2}+m_{3}\right)} .
\end{aligned}
$$

Secondly, we will do the following two steps.

Step 1. We will show that $z\left(t_{1}\right) \geq m_{3}$ for some $t_{1}>0$ by contradiction.

Suppose that $z(t)<m_{3}$ for all positive values of $t$. From the second equation of (5) and (6), we can see that

$$
\begin{aligned}
\frac{\mathrm{d} w}{\mathrm{~d} t} & =a_{7} f_{1}(z)-\frac{a_{8} f_{1}(z) w}{k_{5}+f_{1}(z)}-\frac{a_{9} f_{2}(z) w}{k_{6}+f_{2}(z)}-b_{4} w, \quad t \neq n T, \\
& \geq \frac{a_{1} a_{7} b_{2}\left(k_{2}+z\right)}{A\left(k_{1}+z\right)\left(k_{2}+z\right)}+\frac{a_{2} a_{3} a_{7}\left(k_{1}+z\right)}{A\left(k_{1}+z\right)\left(k_{2}+z\right)}-C w, \\
& \geq \frac{a_{1} a_{7} b_{2} k_{2}+a_{2} a_{3} a_{7} k_{1}}{A\left(k_{1}+m_{3}\right)\left(k_{2}+m_{3}\right)}-C w, \\
w\left(t^{+}\right) & =w(t)+\mu, \quad t=n T .
\end{aligned}
$$

Let us consider the following comparison system: 


$$
\begin{aligned}
\frac{\mathrm{d} P}{\mathrm{~d} t} & =\frac{a_{1} a_{7} b_{2} k_{2}+a_{2} a_{3} a_{7} k_{1}}{A\left(k_{1}+m_{3}\right)\left(k_{2}+m_{3}\right)}-C P, \quad t \neq n T, \\
P\left(t^{+}\right) & =P(t)+\mu, \quad t=n T, \\
P\left(0^{+}\right) & =w\left(0^{+}\right) .
\end{aligned}
$$

A periodic solution of (29) can then be obtained as follows:

$$
\widetilde{P}(t) \equiv \frac{\mu e^{-C(t-n T)}}{1-e^{-C T}}+\frac{a_{1} a_{7} b_{2} k_{2}+a_{2} a_{3} a_{7} k_{1}}{C A\left(k_{1}+m_{3}\right)\left(k_{2}+m_{3}\right)}, \quad t \in(n T,(n+1) T],
$$

with $\widetilde{P}\left(0^{+}\right) \equiv \mu / 1-e^{-C T}+B / C$. Therefore, the positive solution of (29) is

$$
P(t)=\left(P\left(0^{+}\right)-\frac{a_{1} a_{7} b_{2} k_{2}+a_{2} a_{3} a_{7} k_{1}}{C A\left(k_{1}+m_{3}\right)\left(k_{2}+m_{3}\right)}-\frac{\mu}{1-e^{-C T}}\right) e^{-C T}+\widetilde{P}(t) .
$$

Note that $f_{1}\left(m_{3}\right)=\left(a_{1} b_{2} / A\left(k_{1}+m_{3}\right)\right)+\left(a_{2} a_{3} / A\left(k_{2}+\right.\right.$ $\left.\left.m_{3}\right)\right)$ and $f_{2}\left(m_{3}\right)=\left(a_{3} / b_{2}\left(k_{2}+m_{3}\right)\right)+\left(a_{4} / b_{2}\right) f_{1}\left(m_{3}\right)$.

From the first equation of reduced system (5), we have

$$
\begin{aligned}
\frac{\mathrm{d} z}{\mathrm{~d} t} & =\left[\frac{a_{5} f_{1}(z)}{\left(k_{3}+f_{1}^{2}(z)\right)}+\frac{a_{6} f_{2}(z)}{\left(k_{4}+f_{2}^{2}(z)\right)}\right] z w-b_{3} z \\
& \geq\left[\left(\frac{a_{5} f_{1}\left(m_{3}\right)}{\left(k_{3}+f_{1}^{2}\left(m_{3}\right)\right)}+\frac{a_{6} f_{2}\left(m_{3}\right)}{\left(k_{4}+f_{2}^{2}\left(m_{3}\right)\right)}\right) w-b_{3}\right] z \\
& =\left(\widehat{M}_{1} w-b_{3}\right) z .
\end{aligned}
$$

According to the comparison theorem [45], we obtain $w(t) \geq P(t)$. It follows that

$$
\widetilde{P}(t)-\varepsilon_{1}<P(t) \leq w(t), \quad t \neq n T, t \geq T_{1},
$$

for some $T_{1}>0$ and for $\varepsilon_{1}>0$ which is small enough.

Hence,

$$
\begin{aligned}
\frac{\mathrm{d} z}{\mathrm{~d} t} & \geq\left(\widehat{M}_{1}\left(\widetilde{P}(t)-\varepsilon_{1}\right)-b_{3}\right) z, \quad t \neq n T, t \geq T_{1}, \\
z\left(t^{+}\right) & =(1-\rho) z(t), \quad t=n T, t \geq T_{1} .
\end{aligned}
$$

Suppose that $n \in Z_{+}$and $N T \geq T_{1}$. Then, by integration over $(n T,(n+1) T], n \geq N$, we obtain

$$
\begin{aligned}
z((n+1)) T & \geq z(n T)(1-\rho) e^{\left(\int_{n T}^{(n+1) T}\left(\widehat{M}_{1}\left(\widetilde{P}(t)-\varepsilon_{1}\right)-b_{3}\right) \mathrm{d} t\right)} \\
& =z(n T)(1-\rho) e^{\left(\left(\widehat{M}_{1} \widehat{M}_{2}-\widehat{M}_{1} \varepsilon_{1}-b_{3}\right) T+\left(\widehat{M}_{1} \mu / C\right)\right)} \\
& =z(n T) \eta,
\end{aligned}
$$

where $\eta \equiv(1-\rho) e^{\left(\left(\widehat{M}_{1} \widehat{M}_{2}-\widehat{M}_{1} \varepsilon_{1}-b_{3}\right) T+\left(\widehat{M}_{1} \mu / C\right)\right)}$.

We can see that

$$
\ln \eta=\ln (1-\rho)+\left(\left(\widehat{M}_{1} \widehat{M}_{2}-\widehat{M}_{1} \varepsilon_{1}-b_{3}\right) T+\frac{\widehat{M}_{1} \mu}{C}\right) .
$$

Then, for a positive constant $\varepsilon_{1}$ which is sufficiently small, we have

$$
\begin{aligned}
\ln \eta & \approx \ln (1-\rho)+\left(\left(\widehat{M}_{1} \widehat{M}_{2}-\widehat{M}_{1} \varepsilon_{1}-b_{3}\right) T+\frac{\widehat{M}_{1} \mu}{C}\right) \\
& >\ln (1-\rho)+\left(\widehat{M}_{1} \widehat{M}_{2}-b_{3}\right) T \\
& =\left(\widehat{M}_{1} \widehat{M}_{2}-b_{3}\right) T-\ln \left(\frac{1}{1-\rho}\right) .
\end{aligned}
$$

Therefore, if (19) and (30) hold, then a small positive constant $m_{3}$ can be chosen so that $\ln \eta>0$, and hence $\eta>1$. It follows that $z((n+k) T) \geq z(n T) \eta^{k} \longrightarrow \infty$ when $k \longrightarrow \infty$ which contradicts the boundedness of $z(t)$. Hence, there exists $t_{1}>0$ such that $z\left(t_{1}\right) \geq m_{3}$.

Step 2. If $z(t) \geq m_{3}$ for all $t \geq t_{1}$, then the proof is complete; otherwise, there exists $t>t_{1}$ such that $z(t)<m_{3}$ and we then let $t^{*}=\inf \left\{z(t)<m_{3}\right\}$, so that the following two possible cases are obtained.

Case 1. There exists $n_{1} \in Z_{+}$, such that $t^{*}=n_{1} T$. It follows that for $t \in\left(t_{1}, t^{*}\right], z(t) \geq m_{3}$, and we obtain $z(t)=m_{3}$ by the continuity of $z(t)$. When $t$ is large enough, $z(t)$ and $w(t)$ are both bounded above by a positive constant $M$ and $w(t)$ is also bounded below by a positive constant $m_{1}$ which imply that we can choose positive constants $M^{\prime}$ and $m^{\prime}$ for which $z(t)<M^{\prime}$ and $m_{1}^{\prime}<w(t)<M^{\prime}$ such that

$$
m_{1}^{\prime}<\frac{b_{3}}{\widehat{M}_{1}}
$$

with

$$
\left|w\left(t^{*+}\right)-\frac{a_{1} a_{7} b_{2} k_{2}+a_{2} a_{3} a_{7} k_{1}}{C A\left(k_{1}+m_{3}\right)\left(k_{2}+m_{3}\right)}-\frac{\mu}{1-e^{-C T}}\right|-\mu<M_{1}^{\prime} .
$$

We also choose $n_{2}, n_{3} \in Z_{+}$that satisfy the following conditions:

$$
n_{2} T>\frac{1}{B} \ln \left(\frac{M^{\prime}+\mu}{\varepsilon_{1}}\right)
$$

and

$$
(1-\rho)^{n_{2}} e^{\eta_{1}\left(\left(n_{2}+1\right) T\right)} \eta^{n_{3}}>1,
$$

where

$$
\eta_{1} \equiv \widehat{M}_{1} m_{1}^{\prime}-b_{3}<0 .
$$

Here, we let $T^{\prime}=n_{2} T+n_{3} T$. We claim that there exists a constant $t_{2} \in\left(t^{*}, t^{*}+T^{\prime}\right]$ in which $z\left(t_{2}\right)>m_{3}$. Otherwise, by considering (38) with $P\left(t^{*+}\right)=w\left(t^{*+}\right)$, for $t \in(n T,(n+$ 1)T] and $n_{1} \leq n \leq n_{1}+n_{2}+n_{3}$, we obtain 


$$
P(t)=\left(P\left(t^{*+}\right)-\frac{a_{1} a_{7} b_{2} k_{2}+a_{2} a_{3} a_{7} k_{1}}{C A\left(k_{1}+m_{3}\right)\left(k_{2}+m_{3}\right)}-\frac{\mu}{1-e^{-C T}}\right) e^{-C\left(t-t^{*}\right)}+\widetilde{P}(t) .
$$

For $n_{2} T \leq t-t^{*} \leq T^{\prime}$

$$
\begin{aligned}
|P(t)-\widetilde{P}(t)| & =\left|P\left(t^{*+}\right)-\frac{a_{1} a_{7} b_{2} k_{2}+a_{2} a_{3} a_{7} k_{1}}{C A\left(k_{1}+m_{3}\right)\left(k_{2}+m_{3}\right)}-\frac{\mu}{1-e^{-C T}}\right| e^{-C\left(t-t^{*}\right)} \\
& =\left|w\left(t^{*+}\right)-\frac{a_{1} a_{7} b_{2} k_{2}+a_{2} a_{3} a_{7} k_{1}}{C A\left(k_{1}+m_{3}\right)\left(k_{2}+m_{3}\right)}-\frac{\mu}{1-e^{-C T}}\right| e^{-C\left(t-t^{*}\right)} \\
& <\left(M^{\prime}+\mu\right) e^{-C\left(t-t^{*}\right)} \\
& <\left(M^{\prime}+\mu\right) e^{-B n_{2} T} \\
& <\varepsilon_{1} .
\end{aligned}
$$

It follows that

$$
\widetilde{P}(t)-\varepsilon_{1}<P(t)<w(t) .
$$

Similar to Step 1, we then obtain

$$
\begin{aligned}
z\left(t^{*}+T^{\prime}\right) & =z\left(n_{1} T+n_{2} T+n_{3} T\right) \\
& \geq z\left(t^{*}+n_{2} T\right) \eta^{n_{3}}
\end{aligned}
$$

According to the first equation of (5),

$$
\begin{aligned}
\frac{\mathrm{d} z}{\mathrm{~d} t} & =\left[\frac{a_{5} f_{1}(z)}{\left(k_{3}+f_{1}^{2}(z)\right)}+\frac{a_{6} f_{2}(z)}{\left(k_{4}+f_{2}^{2}(z)\right)}\right] z w-b_{3} z, \quad t \neq n T \\
& \geq\left(\widehat{M}_{1} w-b_{3}\right) z \\
& =\left(\widehat{M}_{1} m_{1}^{\prime}-b_{3}\right) z \\
& =\eta_{1} z, \\
z\left(t^{*}\right) & =(1-\rho) z(t), \quad t=n T .
\end{aligned}
$$

By integrating (54) over $\left[t^{*}, t^{*}+n_{2} T\right]$, it follows that

$$
\begin{array}{r}
z\left(t^{*}+n_{2} T\right) \geq z\left(t^{*}\right) e^{\eta_{1}\left(n_{2} T\right)} \\
\geq z\left(t^{*}\right)(1-\rho) e^{\eta_{1}\left(n_{2} T\right)} \\
\geq m_{3}(1-\rho)^{n_{2}} e^{\eta_{1}\left(n_{2} T\right)} \\
\geq m_{3}(1-\rho)^{n_{2}} e^{\eta_{1}\left(\left(n_{2}+1\right) T\right)} .
\end{array}
$$

Therefore, we obtain

$$
\begin{aligned}
& z\left(t^{*}+T^{\prime}\right) \geq z\left(t^{*}+n_{2} T\right) \eta^{n_{3}} \\
& \geq m_{3}(1-\rho)^{n_{2}} e^{\eta_{1}\left(\left(n_{2}+1\right) T\right)} \eta^{n_{3}} \\
& >m_{3},
\end{aligned}
$$

which is a contradiction, and hence, $z\left(t_{2}\right)>m_{3}$ for some $t_{2} \in\left(t^{*}, t^{*}+T^{\prime}\right]$

Next, we let $\tilde{t}=\inf _{t t^{*}}\left\{z(t)>m_{3}\right\}$. It follows that $z(t)<m_{3}$ for $t \in\left(t^{*}, \tilde{t}\right)$, and we can obtain $z(\widetilde{t})=m_{3}$ by the continuity of $z(t)$. Then, $l \in Z_{+}$is chosen where $l \leq n_{2}+n_{3}$ and $t^{*}+l T \geq \tilde{t}$. Suppose $t \in\left(t^{*}+(l-1) T, t^{*}+l T\right]$. From (54), we obtain

$$
\begin{aligned}
z(t) & \geq z\left(t^{*}+(l-1) T\right)(1-\rho)^{l-1} e^{\eta_{1}\left(t-\left(t^{*}+(l-1) T\right)\right)} \\
& \geq m_{3}(1-\rho)^{l} e^{\left(\eta_{1}\left(t-t^{*}\right)\right)} \\
& \geq m_{3}(1-\rho)^{n_{2}+n_{3}} e^{\eta_{1} l T} \\
& \geq m_{3}(1-\rho)^{n_{2}+n_{3}} e^{\eta_{1}\left(n_{2}+n_{3}\right) T} .
\end{aligned}
$$

Since $\eta_{1}$ is negative and $l<n_{2}+n_{3}$, we let

$$
\overline{m_{2}}=m_{3}(1-\rho)^{n_{2}+n_{3}} e^{\eta_{1}\left(n_{2}+n_{3}\right) T}
$$

so that $z(t) \geq \overline{m_{2}}$ for $\left(t^{*}, \widetilde{t}\right)$. We can use $\tilde{t}$ instead of $t^{*}$, the proof can be proceeded in the same manner and consequently, we will obtain $z(t) \geq \overline{m_{2}}$ for sufficiently large $t$.

Case 2. $t^{*} \neq n T$ for all $n \in Z_{+}$. Then, for $t \in\left[t_{1}, t^{*}\right)$, we have $z(t) \geq m_{3}$ and $z\left(t^{*}\right)=m_{3}$. We assume that there exists $n_{1}^{\prime} \in Z_{+}$such that $t^{*} \in\left(n_{1}^{\prime} T,\left(n_{1}^{\prime}+1\right) T\right)$. This leads to the following two possible subcases:

Case 2.1: for all $t \in\left(t^{*},\left(n_{1}^{\prime}+1\right) T\right], z(t) \leq m_{3}$. If there exists a constant $t_{2}^{\prime} \in\left[\left(n_{1}^{\prime}+1\right) T,\left(n_{1}^{\prime}+1\right) T+T^{\prime}\right]$, then we can claim that $z\left(t_{2}^{\prime}\right)>m_{3}$. Otherwise, let us consider (38) with $P\left(\left(n_{1}^{\prime}+1\right) T^{+}\right)=w\left(\left(n_{1}^{\prime}+1\right) T^{+}\right)$. For $t \in(n T,(n+1) T], n_{1}^{\prime}+1 \leq n \leq n_{1}^{\prime}+1+n_{2}+n_{3}$, it follows that

$$
P(t)=\left(P\left(\left(n_{1}^{\prime}+1\right) T^{+}\right)-\frac{a_{1} a_{7} b_{2} k_{2}+a_{2} a_{3} a_{7} k_{1}}{C A\left(k_{1}+m_{3}\right)\left(k_{2}+m_{3}\right)}-\frac{\mu}{1-e^{-C T}}\right) e^{-C\left(t-\left(n_{1}^{\prime}+1\right) T\right)}+\widetilde{P}(t) .
$$

By a similar argument in Case 1, we have

$$
|P(t)-\widetilde{P}(t)|<\varepsilon_{1},
$$

for $n_{2} T \leq t-t^{*}$. It follows that

$$
\widetilde{P}(t)-\varepsilon_{1}<P(t) \leq w(t) .
$$


Since $n_{2} T \leq\left(n_{1}^{\prime}+1+n_{2}\right) T-t^{*}$, we then have

$$
\begin{aligned}
z\left(\left(n_{1}^{\prime}+1+n_{2}\right) T\right) & \geq z\left(t^{*}\right) e^{\eta_{1}\left(\left(n_{1}^{\prime}+1+n_{2}\right) T-t^{*}\right)} \\
& \geq z\left(t^{*}\right)(1-\rho)^{n_{2}} e^{\eta_{1}\left(\left(n_{1}^{\prime}+1+n_{2}\right) T-t^{*}\right)} \\
& \geq m_{3}(1-\rho)^{n_{2}} e^{\eta_{1}\left(n_{2}+1\right) T} .
\end{aligned}
$$

Thus,

$$
\begin{aligned}
z\left(\left(n_{1}^{\prime}+1+n_{2}+n_{3}\right) T\right) & \geq z\left(\left(n_{1}^{\prime}+1+n_{2}\right) T\right) \eta^{n_{3}} \\
& \geq m_{3}(1-\rho)^{n_{2}} e^{\eta_{1}\left(n_{2}+1\right) T} \eta^{n_{3}} \\
& >m_{3},
\end{aligned}
$$

which is a contradiction. Therefore, we obtain $z\left(t_{2}^{\prime}\right)>m_{3}$ for some $t_{2}^{\prime} \in\left[\left(n_{1}^{\prime}+1\right) T,\left(n_{1}^{\prime}+1\right) T+T^{\prime}\right]$.

Next, we assume that $\bar{t}=\inf _{t>t^{*}}\left\{z(t)>m_{3}\right\}$. Then, we have $z(t) \leq m_{3}$ for $t \in\left[t^{*}, \bar{t}\right)$ and $z(\bar{t})=m_{3}$. Suppose that $t \in\left(n_{1}^{\prime} T+\left(l^{\prime}-1\right) T, n_{1}^{\prime} T+l^{\prime} T\right]$ and a positive number $l^{\prime}$ is chosen for which $l^{\prime} \leq n_{2}+n_{3}+1$. From (54), we obtain

$$
\begin{aligned}
& z(t) \geq z\left(n_{1}^{\prime} T+\left(l^{\prime}-1\right) T\right) e^{\eta_{1}\left(t-\left(n_{1}^{\prime} T+\left(l^{\prime}-1\right) T\right)\right)} \\
& z\left(n_{1}^{\prime} T+\left(l^{\prime}-1\right) T\right) \geq z\left(t^{*}\right) e^{\eta_{1}\left(n_{1}^{\prime} T+\left(l^{\prime}-1\right) T-t^{*}\right)} .
\end{aligned}
$$

Therefore,

$$
\begin{aligned}
z(t) & \geq z\left(n_{1}^{\prime} T+\left(l^{\prime}-1\right) T\right)(1-\rho)^{l-1} e^{\eta_{1}\left(t-\left(n_{1}^{\prime} T+\left(l^{\prime}-1\right) T\right)\right)} \\
& \geq z\left(t^{*}\right)(1-\rho)^{l-1} e^{\eta_{1}\left(t-t^{*}\right)} \\
& \geq m_{3}(1-\rho)^{l^{\prime}-1} e^{\eta_{1}\left(t-t^{*}\right)} .
\end{aligned}
$$

Since $n_{1}^{\prime} T+\left(l^{\prime}-1\right) T<t \leq n_{1}^{\prime} T+l^{\prime} T$ and $n_{1}^{\prime} T<t^{*}<\left(n_{1}^{\prime}\right.$ $+1) T$, we then obtain $t-t^{*} \leq l^{\prime} T<\left(n_{2}+n_{3}+1\right) T$. Thus,

$$
z(t) \geq m_{3}(1-\rho)^{n_{2}+n_{3}} e^{\eta_{1}\left(n_{2}+n_{3}+1\right) T} .
$$

We let

$$
m_{2}=m_{3}(1-\rho)^{n_{2}+n_{3}} e^{\eta_{1}\left(n_{2}+n_{3}+1\right) T} .
$$

It follows that $z(t) \geq m_{2}$ for $t \in\left(t^{*}, \bar{t}\right)$. We can use $\bar{t}$ instead of $t^{*}$, the proof can be proceeded in the same manner, and consequently, we will obtain $z(t) \geq m_{2}$ for sufficiently large $t$.

Case 2.2: there exists $t^{\prime \prime} \in\left(t^{*},\left(n_{1}^{\prime}+1\right) T\right]$ such that $z\left(t^{\prime \prime}\right)>m_{3}$. We assume that $\underline{t}=\inf _{t>t^{*}}\left\{z(t)>m_{3}\right\}$. Then, we have $z(t)<m_{3}$ for $t \in\left[t^{*}, \underline{t}\right)$, and $z(\underline{t})=m_{3}$. Since $t<\left(n_{1}^{\prime}+1\right) T<t^{*}+T$, for $t \in\left[t^{*}, \underline{t}\right)$, we obtain the following:

$$
\begin{aligned}
z(t) & \geq z\left(t^{*}\right) e^{\eta_{1}\left(t-t^{*}\right)} \\
& \geq m_{3} e^{\eta_{1} T} \\
& =m_{2} .
\end{aligned}
$$

Since $z(\underline{t}) \geq m_{3}$, we can proceed the proof in the same manner for $t>\underline{t}$. We then have $z(t) \geq m_{2}$ for $t \geq t_{1}$ because $m_{2}<\overline{m_{2}}<m_{3}$ and the proof is complete.

\section{Periodic Solution: Existence and Stability}

To investigate the possibility of a bifurcation of the nontrivial periodic solution to systems (5) and (6) near $(0, \widetilde{w}(t))$, we first interchange the variables of $z$ and $w$ for convenience on computation. In what follows, let us consider the following system instead:

$$
\left.\begin{array}{l}
\frac{\mathrm{d} z}{\mathrm{~d} t}=a_{7} f_{1}(w)-\frac{a_{8} f_{1}(w) z}{k_{5}+f_{1}(w)}-\frac{a_{9} f_{2}(w) z}{k_{6}+f_{2}(w)}-b_{4} z, \\
\frac{\mathrm{d} w}{\mathrm{~d} t}=\left[\frac{a_{5} f_{1}(w)}{\left(k_{3}+f_{1}^{2}(w)\right)}+\frac{a_{6} f_{2}(w)}{\left(k_{4}+f_{2}^{2}(w)\right)}\right] z w-b_{3} w,
\end{array}\right\} t \neq n T,
$$

with

$$
\left.\begin{array}{l}
\Delta z(t)=\mu, \\
\Delta w(t)=-\rho w(t),
\end{array}\right\} t=n T
$$

Let

$$
\begin{gathered}
G_{1}(z, w)=a_{7} f_{1}(w)-\frac{a_{8} f_{1}(w) z}{k_{5}+f_{1}(w)}-\frac{a_{9} f_{2}(w) z}{k_{6}+f_{2}(w)}-b_{4} z \\
G_{2}(z, w)=\left[\frac{a_{5} f_{1}(w)}{\left(k_{3}+f_{1}^{2}(w)\right)}+\frac{a_{6} f_{2}(w)}{\left(k_{4}+f_{2}^{2}(w)\right)}\right] z w-b_{3} w .
\end{gathered}
$$

Note that

$f_{1}(w)=\frac{a_{1} b_{2}}{A\left(k_{1}+w\right)}+\frac{a_{2} a_{3}}{A\left(k_{2}+w\right)}$

$f_{1}^{\prime}(w)=-\left(\frac{a_{1} b_{2}}{A\left(k_{1}+w\right)^{2}}+\frac{a_{2} a_{3}}{A\left(k_{2}+w\right)^{2}}\right)$

$f_{2}(w)=\frac{a_{3}}{b_{2}\left(k_{2}+w\right)}+\frac{a_{1} a_{4}}{A\left(k_{1}+w\right)}+\frac{a_{2} a_{3} a_{4}}{A b_{2}\left(k_{2}+w\right)}$

$f_{2}^{\prime}(w)=-\left(\frac{a_{3}}{b_{2}\left(k_{2}+w\right)^{2}}+\frac{a_{1} a_{4}}{A\left(k_{1}+w\right)^{2}}+\frac{a_{2} a_{3} a_{4}}{A b_{2}\left(k_{2}+w\right)^{2}}\right)$.

According to Lakmeche and Ario [45], the following notations are used: 


$$
\begin{aligned}
\frac{\partial \Phi_{1}}{\partial w} & =\frac{\partial \Phi_{2}}{\partial z}=0, \\
\frac{\partial \Phi_{1}}{\partial z} & =1, \\
\frac{\partial \Phi_{2}}{\partial w} & =1-\rho, \\
\frac{\partial^{2} \Phi_{1}}{\partial z \partial w} & =\frac{\partial^{2} \Phi_{2}}{\partial z \partial w}=\frac{\partial^{2} \Phi_{2}}{\partial w^{2}}, \\
\Theta_{1}(z, w) & =z+\mu, \\
\Theta_{2}(z, w) & =(1-\rho) w, \\
\zeta(t) & =(\widetilde{w}(t), 0)^{T}, \\
S_{0} & =\left(\widetilde{w}\left(\tau_{0}\right), 0\right)^{T}, \\
\tau_{0} & =T_{\max } .
\end{aligned}
$$

$$
\begin{aligned}
& \frac{\partial G_{1}(\zeta(r))}{\partial z}=-\frac{a_{8} f_{1}(0)}{k_{5}+f_{1}(0)}-\frac{a_{9} f_{2}(0)}{k_{6}+f_{2}(0)}-b_{4}=-C \\
& \frac{\partial G_{2}(\zeta(r))}{\partial w}=\left[\frac{a_{5} f_{1}(0)}{k_{3}+f_{1}^{2}(0)}+\frac{a_{6} f_{2}(0)}{k_{4}+f_{2}^{2}(0)}\right] \widetilde{w}(r)-b_{3}=D \widetilde{w}(r)-b_{3} \text {, } \\
& \frac{\partial G_{1}(\zeta(r))}{\partial w}=a_{7} f_{1}^{\prime}(0)-a_{8} \widetilde{w}(r)\left(\frac{f_{1}^{\prime}(0)}{k_{5}+f_{1}(0)}-\frac{f_{1}^{\prime}(0) f_{1}(0)}{\left(k_{5}+f_{1}(0)\right)^{2}}\right)-a_{9} \widetilde{w}(r)\left(\frac{f_{2}^{\prime}(0)}{k_{6}+f_{2}(0)}-\frac{f_{2}^{\prime}(0) f_{2}(0)}{\left(k_{6}+f_{2}(0)\right)^{2}}\right) \text {, } \\
& \frac{\partial G_{2}(\zeta(r))}{\partial z \partial w}=\frac{a_{5} f_{1}(0)}{k_{3}+f_{1}^{2}(0)}+\frac{a_{6} f_{2}(0)}{k_{4}+f_{2}^{2}(0)}=D \\
& \frac{\partial G_{2}(\zeta(r))}{\partial w^{2}}=2 a_{5} \widetilde{w}(r)\left(\frac{f_{1}^{\prime}(0)}{k_{3}+f_{1}^{2}(0)}-\frac{2 f_{1}^{\prime}(0) f_{1}^{2}(0)}{\left(k_{3}+f_{1}^{2}(0)\right)^{2}}\right)+2 a_{6} \widetilde{w}(r)\left(\frac{f_{2}^{\prime}(0)}{k_{4}+f_{2}^{2}(0)}-\frac{2 f_{2}^{\prime}(0) f_{2}^{2}(0)}{\left(k_{4}+f_{2}^{2}(0)\right)^{2}}\right), \\
& \frac{\partial \Phi_{1}\left(\tau_{0}, S_{0}\right)}{\partial \tau}=\frac{\partial \widetilde{w}\left(\tau_{0}, S_{0}\right)}{\partial t}=\frac{-C \mu \exp \left(-C \tau_{0}\right)}{1-\exp \left(-C \tau_{0}\right)}<0, \\
& \frac{\partial \Phi_{1}\left(\tau_{0}, S_{0}\right)}{\partial z}=\exp \left(\int_{0}^{\tau_{0}} \frac{\partial G_{1}(\zeta(r))}{\partial z} \mathrm{~d} r\right)=\exp \left(-C \tau_{0}\right)>0, \\
& \frac{\partial \Phi_{1}\left(\tau_{0}, S_{0}\right)}{\partial w}=\int_{0}^{\tau_{0}} \exp \left(\frac{\partial G_{1}(\zeta(r))}{\partial z} \mathrm{~d} r\right) \frac{\partial G_{1}(\zeta(\nu))}{\partial w} \exp \left(\int_{0}^{\nu} \frac{\partial G_{2}(\zeta(r))}{\partial w} \mathrm{~d} r\right) \mathrm{d} \nu \\
& =\int_{0}^{\tau_{0}} \exp \left(-C\left(\tau_{0}-\nu\right)\right) \times\left[a_{7} f_{1}^{\prime}(0)-a_{8} \widetilde{w}(\nu)\left(\frac{f_{1}^{\prime}(0)}{k_{5}+f_{1}(0)}-\frac{f_{1}^{\prime}(0) f_{1}(0)}{\left(k_{5}+f_{1}(0)\right)^{2}}\right)\right. \\
& \left.-a_{9} \widetilde{w}(\nu)\left(\frac{f_{2}^{\prime}(0)}{k_{6}+f_{2}(0)}-\frac{f_{2}^{\prime}(0) f_{2}(0)}{\left(k_{6}+f_{2}(0)\right)^{2}}\right)\right] \times \exp \left(\int_{0}^{v}\left(\left(D \widetilde{w}(r)-b_{3}\right) \mathrm{d} r\right)\right) \mathrm{d} \nu \\
& \frac{\partial \Phi_{2}\left(\tau_{0}, S_{0}\right)}{\partial w}=\exp \left(\int_{0}^{\tau_{0}} \frac{\partial G_{2}(\zeta(r))}{\partial w} \mathrm{~d} r\right)=\exp \left(\int_{0}^{\tau_{0}}\left(D \widetilde{w}(r)-b_{3}\right) \mathrm{d} r\right)>0, \\
& \frac{\partial^{2} \Phi_{2}\left(\tau_{0}, S_{0}\right)}{\partial z \partial w}=\int_{0}^{\tau_{0}} \exp \left(\int_{v}^{\tau_{0}} \frac{\partial G_{2}(\zeta(r))}{\partial w} \mathrm{~d} r\right) \frac{\partial^{2} G_{2}(\zeta(\nu))}{\partial z \partial w} \exp \left(\int_{0}^{v} \frac{\partial G_{2}(\zeta(r))}{\partial w} \mathrm{~d} r\right) \mathrm{d} v \\
& =\int_{0}^{\tau_{0}} \exp \left(\int_{v}^{\tau_{0}}\left(D \widetilde{w}(r)-b_{3}\right) \mathrm{d} r\right) D \exp \left(\int_{0}^{v}\left(D \widetilde{w}(r)-b_{3}\right) \mathrm{d} r\right) \mathrm{d} v>0,
\end{aligned}
$$




$$
\begin{aligned}
\frac{\partial^{2} \Phi_{2}\left(\tau_{0}, S_{0}\right)}{\partial w^{2}}= & \int_{0}^{\tau_{0}} \exp \left(\int_{v}^{\tau_{0}} \frac{\partial G_{2}(\zeta(r))}{\partial w} \mathrm{~d} r\right) \frac{\partial^{2} G_{2}(\zeta(v))}{\partial w^{2}} \exp \left(\int_{0}^{v} \frac{\partial G_{2}(\zeta(r))}{\partial w} \mathrm{~d} r\right) \mathrm{d} v \\
& +\int_{0}^{\tau_{0}}\left[\exp \left(\int_{v}^{\tau_{0}} \frac{\partial G_{2}(\zeta(r))}{\partial w} \mathrm{~d} r\right) \frac{\partial^{2} G_{2}(\zeta(r))}{\partial z \partial w}\right] \\
& \times\left[\int_{0}^{v} \exp \left(\int_{\theta}^{v} \frac{\partial G_{1}(\zeta(r))}{\partial z} \mathrm{~d} r\right) \frac{\partial G_{1}(\zeta(\theta))}{\partial w} \exp \left(\int_{0}^{\theta} \frac{\partial G_{2}(\zeta(r))}{\partial w} \mathrm{~d} r\right) \mathrm{d} \theta\right] \mathrm{d} v \\
\frac{\partial^{2} \Phi_{2}\left(\tau_{0}, S_{0}\right)}{\partial w \partial \tau}= & \frac{\partial G_{2}\left(\zeta\left(\tau_{0}\right)\right)}{\partial w} \exp \left(\int_{0}^{\tau_{0}} \frac{\partial G_{2}(\zeta(r))}{\partial w} \mathrm{~d} r\right) \\
= & \left(D \widetilde{w}\left(\tau_{0}\right)-b_{3}\right) \exp \left(\int_{0}^{\tau_{0}}\left(D \widetilde{w}(r)-b_{3}\right) \mathrm{d} r\right) .
\end{aligned}
$$

Now, we can compute where $\tau_{0}$ is the root of $d_{0}^{\prime}=0$ and

$$
\begin{aligned}
d_{0}^{\prime} & =1-\left(\frac{\partial \Theta_{2}}{\partial w} \frac{\partial \Phi_{2}}{\partial w}\right)_{\left(\tau_{0}, S_{0}\right)} \\
& =1-(1-\rho) \exp \left(\int_{0}^{\tau_{0}}\left(D \widetilde{w}(r)-b_{3}\right) \mathrm{d} r\right),
\end{aligned}
$$

$$
\begin{aligned}
& a_{0}^{\prime}=1-\left(\frac{\partial \Theta_{1}}{\partial z} \frac{\partial \Phi_{1}}{\partial z}\right)_{\left(\tau_{0}, S_{0}\right)}=1-\exp \left(-C \tau_{0}\right)>0 \\
& b_{0}^{\prime}=-\left(\frac{\partial \Theta_{1}}{\partial z} \frac{\partial \Phi_{1}}{\partial w}+\frac{\partial \Theta_{1}}{\partial w} \frac{\partial \Phi_{2}}{\partial w}\right)_{\left(\tau_{0}, S_{0}\right)}=-\frac{\partial \Theta_{1}\left(\tau_{0}, S_{0}\right)}{\partial w}, \\
& I=-\frac{\partial^{2} \Theta_{2}}{\partial z \partial w}\left(\frac{\partial \Phi_{1}\left(\tau_{0}, S_{0}\right)}{\partial \tau}+\frac{\partial \Phi_{1}\left(\tau_{0}, S_{0}\right)}{\partial z} \frac{1}{a_{0}^{\prime}} \frac{\partial \Theta_{1}}{\partial z} \frac{\partial \Phi_{1}\left(\tau_{0}, S_{0}\right)}{\partial \tau}\right) \\
& \frac{\partial \Phi_{2}\left(\tau_{0}, S_{0}\right)}{\partial w}-\frac{\partial \Theta_{2}}{\partial w} \\
& \left(\frac{\partial^{2} \Phi_{2}\left(\tau_{0}, S_{0}\right)}{\partial z \partial w} \frac{1}{a_{0}^{\prime}} \frac{\partial \Theta_{1}}{\partial z} \frac{\partial \Phi_{1}\left(\tau_{0}, S_{0}\right)}{\partial \tau}+\frac{\partial \Phi_{2}\left(\tau_{0}, S_{0}\right)}{\partial \tau \partial w}\right) \\
& =-(1-\rho) \\
& \left(\frac{\partial^{2} \Phi_{2}\left(\tau_{0}, S_{0}\right)}{\partial z \partial w} \frac{1}{1-\exp \left(-C \tau_{0}\right)} \frac{\partial \Phi_{1}\left(\tau_{0}, S_{0}\right)}{\partial \tau}+\left(D \widetilde{w}\left(\tau_{0}\right)-b_{3}\right) \exp \left(\int_{0}^{\tau_{0}}\left(D \widetilde{w}(r)-b_{3}\right) d r\right)\right) \\
& =-(1-\rho) \exp \left(\frac{D \mu}{C}+\frac{D B \tau_{0}}{C}-b_{3} \tau_{0}\right) \\
& \left(\frac{-C D \tau_{0} \mu \exp \left(-C \tau_{0}\right)}{\left(1-\exp \left(-C \tau_{0}\right)\right)^{2}}+D \widetilde{w}\left(\tau_{0}\right)-b_{3}\right) \\
& J=-2 \frac{\partial^{2} \Theta_{2}}{\partial z \partial w}\left(-\frac{b_{0}^{\prime}}{a_{0}^{\prime}} \frac{\partial \Phi_{1}\left(\tau_{0}, S_{0}\right)}{\partial z}+\frac{\partial \Phi_{1}\left(\tau_{0}, S_{0}\right)}{\partial w}\right) \frac{\partial \Phi_{2}\left(\tau_{0}, S_{0}\right)}{\partial w}-\frac{\partial^{2} \Theta_{2}}{\partial w^{2}}\left(\frac{\partial \Phi_{2}\left(\tau_{0}, S_{0}\right)}{\partial w}\right)^{2} \\
& +2 \frac{\partial \Phi_{2}}{\partial w} \frac{b_{0}^{\prime}}{a_{0}^{\prime}} \frac{\partial^{2} \Phi_{2}\left(\tau_{0}, S_{0}\right)}{\partial w \partial z}-\frac{\partial \Theta_{2}}{\partial w} \frac{\partial^{2} \Phi_{2}\left(\tau_{0}, S_{0}\right)}{\partial w^{2}} \\
& =2(1-\rho) \frac{b_{0}^{\prime}}{a_{0}^{\prime}} \frac{\partial^{2} \Phi_{2}\left(\tau_{0}, S_{0}\right)}{\partial w \partial z}-(1-\rho) \frac{\partial^{2} \Phi_{2}\left(\tau_{0}, S_{0}\right)}{\partial w^{2}} .
\end{aligned}
$$



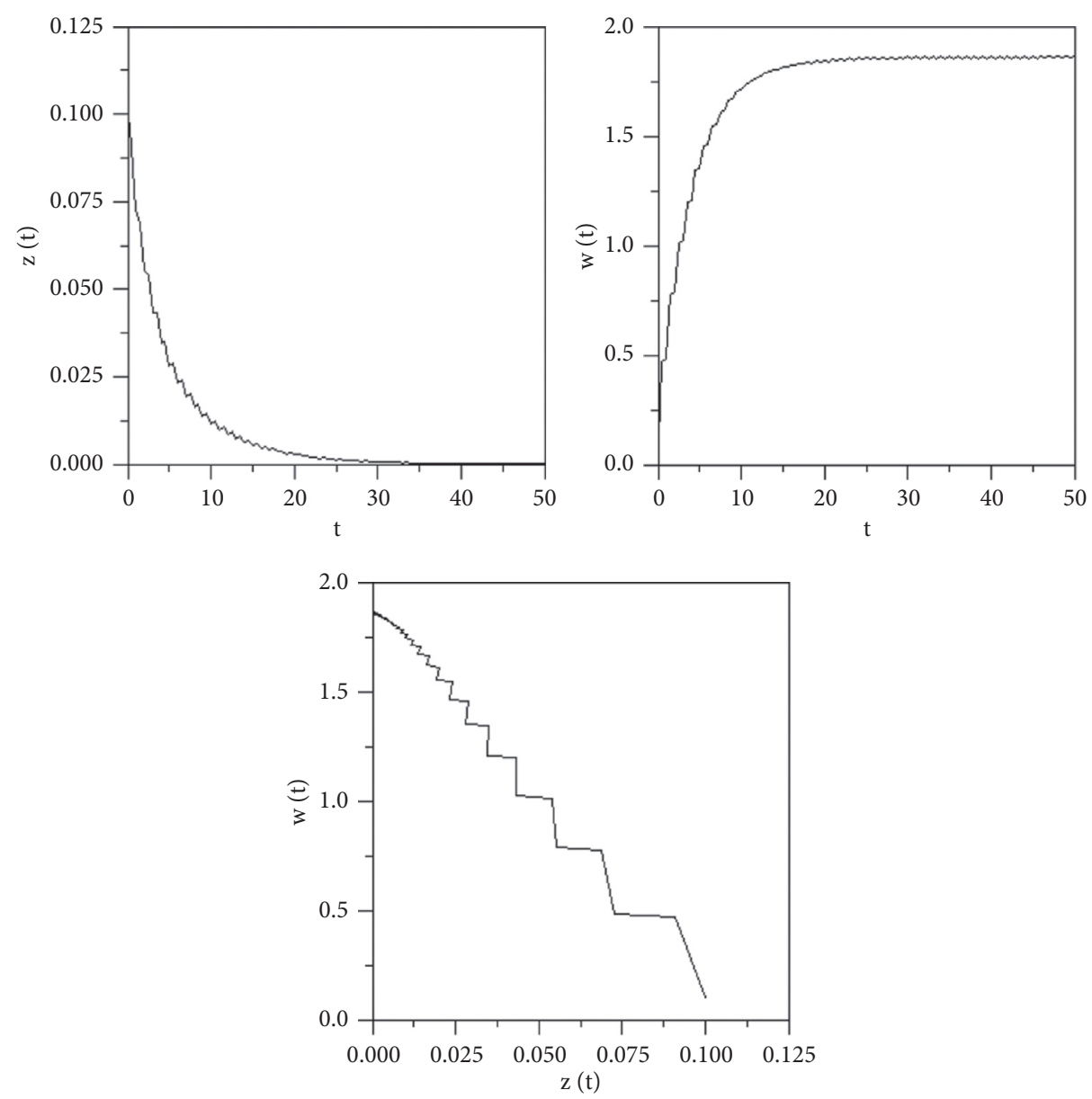

Figure 1: A computer simulation of systems (5) and (6). The solution trajectory approaches oscillatory solution $(0, \widetilde{w}(t))$ when time passes. Here, $\quad a_{1}=0.32, a_{2}=0.58, a_{3}=0.19, a_{4}=0.39, a_{5}=0.1, \quad a_{6}=0.34, a_{7}=0.91, a_{8}=0.7, a_{9}=0.03, \quad b_{1}=0.98, b_{2}=0.68, b_{3}=0.25, b_{4}$ $=0.15, k_{1}=0.56, k_{2}=0.95, k_{3}=0.96, k_{4}=0.99, k_{5}=0.84, k_{6}=0.65, \mu=0.01, \rho=0.2, T=1, z(0)=0.1$, and $w(0)=0.1$ in which all conditions in Theorem 1 are satisfied.

We can see that $I>0$ if

$$
\begin{gathered}
T>\frac{1}{C} \ln \left(1+\frac{\mu C D}{b_{3} C-B D}\right), \\
\mu<\frac{B}{C}-\frac{b_{3}}{D},
\end{gathered}
$$

while $J<0$ provided that (19) and

$$
\begin{gathered}
\frac{B}{C}\left(K_{1}+K_{2}\right)>K_{0}, \\
k_{3}<f_{1}^{2}(0), \\
k_{4}<f_{2}^{2}(0),
\end{gathered}
$$

hold where $K_{0}=a_{7} f_{1}^{\prime}(0), K_{1}=-\left(a_{8} k_{5} f_{1}^{\prime}(0) /\left(k_{5}+f_{1}(0)\right)^{2}\right)$ and $K_{2}=-\left(a_{9} k_{6} f_{2}^{\prime}(0) /\left(k_{6}+f_{2}(0)\right)^{2}\right)$.

This leads to the following theorem according to Lakmeche and Ario [45].
Theorem 3. If (19), (77), and (78) hold, then systems (5) and (6) have a positive periodic solution.

\section{Numerical Simulations}

In this section, numerical simulations are performed to illustrate the theoretical predictions as proved in the previous section.

To illustrate the theoretical prediction in Theorem 1, the parameters in systems (5) and (6) are chosen to satisfy the conditions in (18) and (20) as follows: $a_{1}=0.32, a_{2}$ $=0.58, a_{3}=0.19, a_{4}=0.39, a_{5}=0.1, a_{6}=0.34, a_{7}=0.91, a_{8}$ $=0.7, a_{9}=0.03, b_{1}=0.98, b_{2}=0.68, b_{3}=0.25, b_{4}=0.15, k_{1}$ $=0.56, k_{2}=0.95, k_{3}=0.96, k_{4}=0.99, k_{5}=0.84, k_{6}=0.65, \mu$ $=0.01, \rho=0.2, T=1, z(0)=0.1$, and $w(0)=0.1$. The computer simulation is shown in Figure 1 . We can see that the solution of systems (5) and (6) converges asymptotically to the oscillating solution $(0, \widetilde{w}(t))$ as time passes for which the active osteoblasts are vanished as predicted in Theorem 1.

To illustrate the theoretical prediction in Theorem 2, the parameters in systems (5) and (6) are chosen to satisfy the conditions in (10), (19), and (30) as follows: $a_{1}=0.32, a_{2}$ 

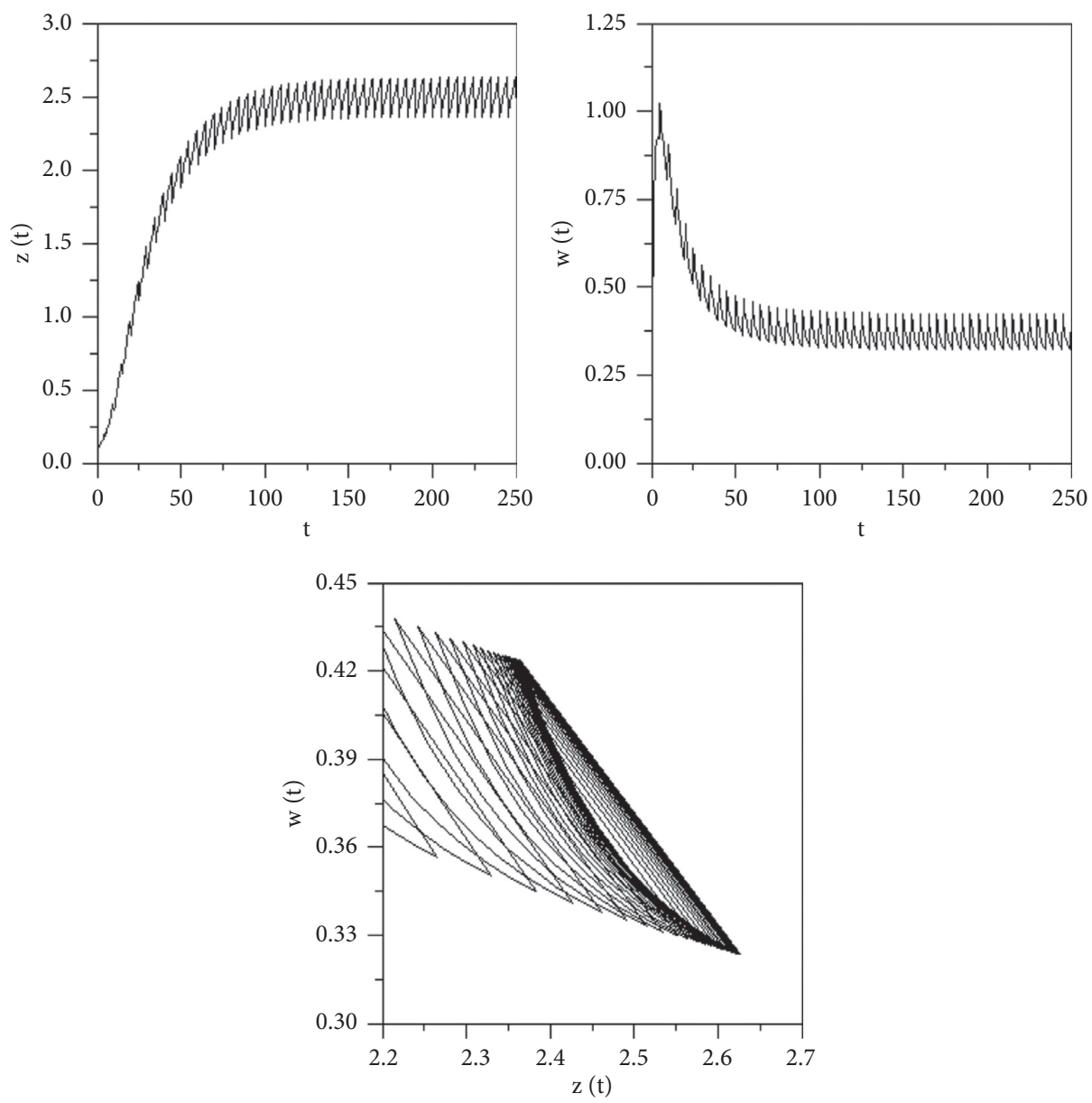

Figure 2: A computer simulation of systems (5) and (6) showing the permanence of the system. Here, $a_{1}=0.32, a_{2}$ $=0.58, a_{3}=0.19, a_{4}=0.39, a_{5}=0.1, a_{6}=0.34, a_{7}=0.91, a_{8}=0.7, a_{9}=0.03, b_{1}=0.98, b_{2}=0.68, b_{3}=0.01, b_{4}=0.5, k_{1}=0.56, k_{2}=0.95$, $k_{3}=0.96, k_{4}=0.99, k_{5}=0.84, k_{6}=0.65, \mu=0.1, \rho=0.1, T=5, z(0)=0.1$, and $w(0)=0.1$ in which all conditions in Theorem 2 are satisfied.

$=0.58, a_{3}=0.19, a_{4}=0.39, a_{5}=0.1, a_{6}=0.34, a_{7}=0.91, a_{8}$ $=0.7, a_{9}=0.03, b_{1}=0.98, b_{2}=0.68, b_{3}=0.01, b_{4}=0.5, k_{1}=$ $0.56, k_{2}=0.95, k_{3}=0.96, k_{4}=0.99, k_{5}=0.84, k_{6}=0.65, \mu$ $=0.1, \rho=0.1, T=5, z(0)=0.1$, and $w(0)=0.1$. The computer simulation is shown in Figure 2. We can see that the solution of systems (5) and (6) are bounded above and bounded below, and hence, the system shows permanence as predicted in Theorem 2 .

To illustrate the theoretical prediction in Theorem 3, the parameters in systems (5) and (6) are chosen to satisfy the conditions in (29), (77), (78), and (79)-(81) as follows: $a_{1}=$ $0.32, a_{2}=0.58, a_{3}=0.19, a_{4}=0.39, a_{5}=0.1, a_{6}=0.34, a_{7}$ $=0.91, a_{8}=0.7, a_{9}=0.03, b_{1}=0.98, b_{2}=0.68, b_{3}=0.01, b_{4}$ $=0.5, k_{1}=0.56, k_{2}=0.95, k_{3}=0.96, k_{4}=0.2, k_{5}=0.84, k_{6}$ $=0.65, \mu=0.1, \rho=0.1, T=10, z(0)=0.1$, and $w(0)=0.1$.
The computer simulation is shown in Figure 3. We can see that the solution of systems (5) and (6) exhibits sustained oscillations as predicted in Theorem 3 .

In addition, to illustrate the effect of different periods between each estrogen administration, computer simulations of systems (5) and (6) for $T=1,5,10,15$ when $a_{1}=$ $0.32, a_{2}=0.58, a_{3}=0.19, a_{4}=0.39, a_{5}=0.1, a_{6}=0.34, a_{7}$ $=0.91, a_{8}=0.7, a_{9}=0.03, b_{1}=0.98, b_{2}=0.68, b_{3}=0.25, b_{4}$ $=0.15, k_{1}=0.56, \quad k_{2}=0.95, k_{3}=0.96, k_{4} \quad=0.99, k_{5}$ $=0.84, k_{6}=0.65, \quad \mu=0.01, \rho=0.2, z(0)=0.5$, and $w(0)$ $=0.5$ are as shown in Figure 4 .

As shown in Figure 4, we can see that the period between each estrogen administration $T$ is significant. The smaller value of $T$ yields the better result on the number of osteoclasts and osteoblasts. 

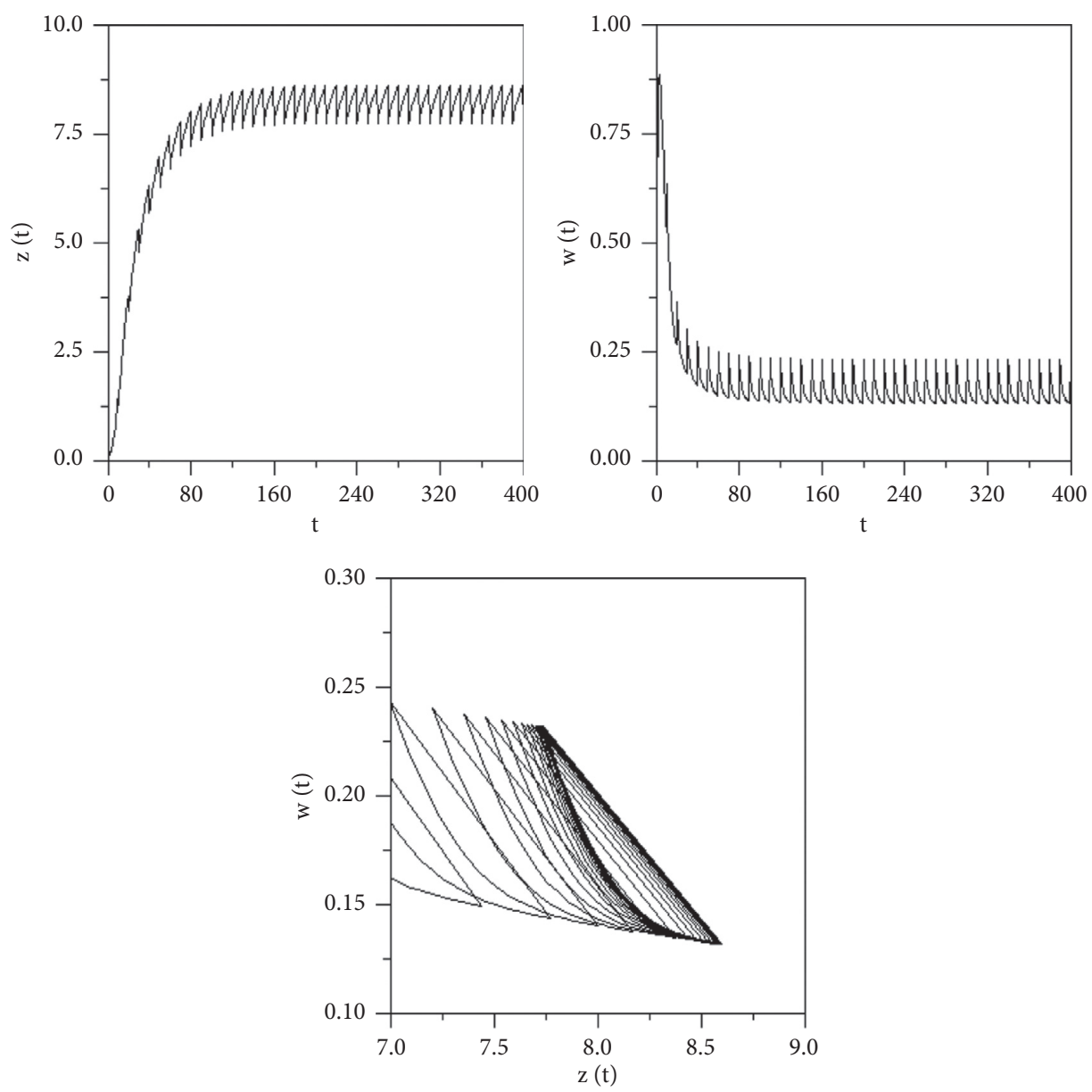

Figure 3: A computer simulation of systems (5) and (6). The solution exhibits sustained oscillations. Here, $a_{1}=0.32, a_{2}=$ $0.58, a_{3}=0.19, a_{4}=0.39, a_{5}=0.1, a_{6}=0.34, a_{7}=0.91, a_{8}=0.7, a_{9}=0.03, b_{1}=0.98, b_{2}=0.68, b_{3}=0.01, b_{4}=0.5, k_{1}=0.56, k_{2}=0.95, k_{3}$ $=0.96, k_{4}=0.2, k_{5}=0.84, k_{6}=0.65, \mu=0.1, \rho=0.1, T=10, z(0)=0.1$, and $w(0)=0.1$, in which all conditions in Theorem 3 are satisfied. 

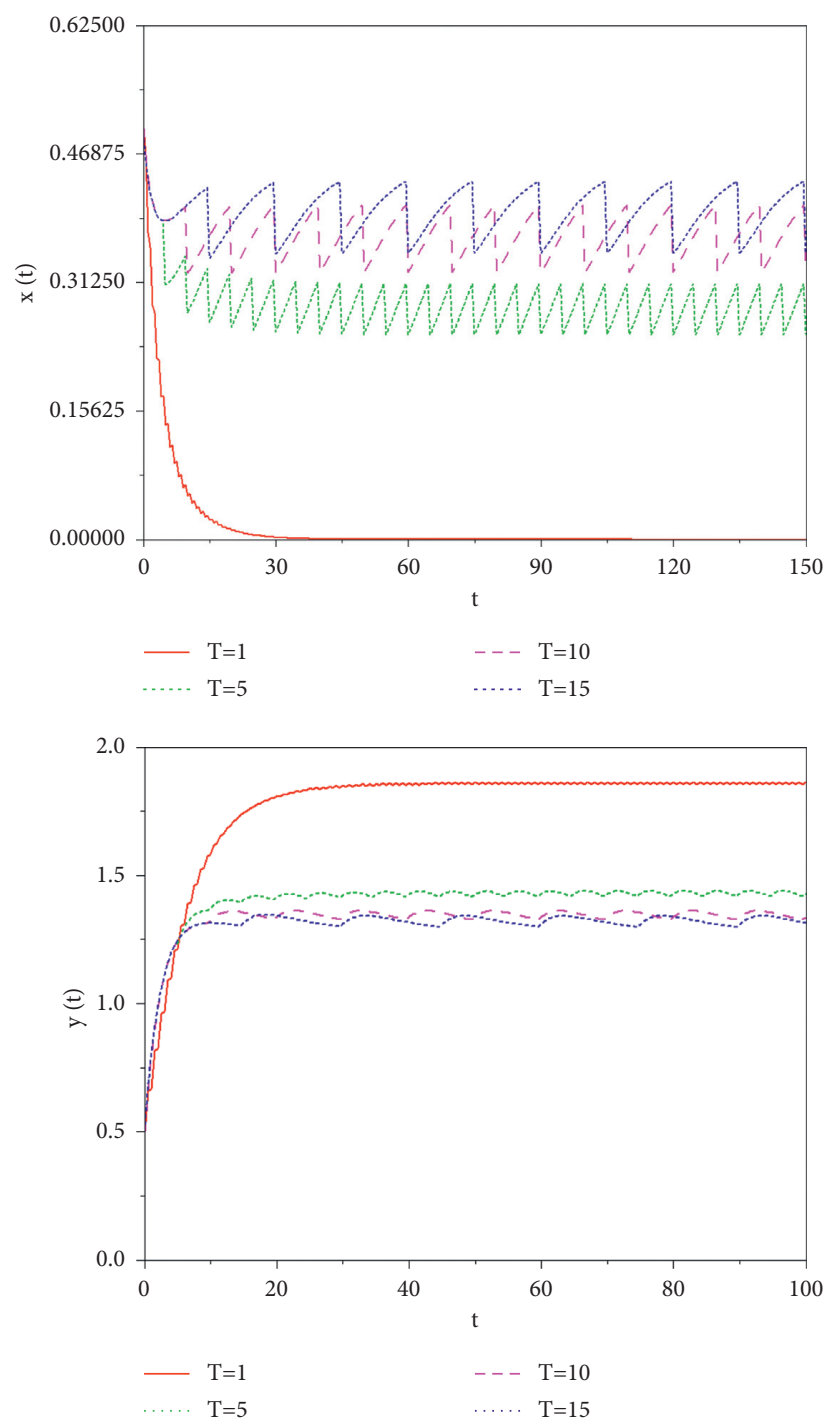

Figure 4: Computer simulations of systems (5) and (6) for $T=1,5,10,15$ when $a_{1}=0.32, a_{2}=0.58, a_{3}=0.19, a_{4}=0.39, a_{5}=0.1, a_{6}=$ $0.34, a_{7}=0.91, \quad a_{8}=0.7, a_{9}=0.03, b_{1}=0.98, b_{2}=0.68, b_{3}=0.25, b_{4}=0.15, k_{1}=0.56, \quad k_{2}=0.95, k_{3}=0.96, \quad k_{4}=0.99, k_{5}=0.84, k_{6}$ $=0.65, \mu=0.01, \rho=0.2, z(0)=0.5$, and $w(0)=0.5$.

\section{Conclusion}

In this paper, an impulsive mathematical model of the process of bone remodeling accounted for bone-resorbing cells, osteoclasts, and bone-forming cells, osteoblasts, is developed in order to investigate the effect of impulsive estrogen supplement. The effects of parathyroid hormone and prolactin are also taken into account. We then apply the Floquet theory and the comparison theorem to derive the conditions in which the periodic solution is locally asymptotically stable. Moreover, the permanence of the system is also investigated as well so that we arrived at the conditions for which the sustained oscillation of the solution is guaranteed. In addition, computer simulations are presented to illustrate the theoretical predictions. The results indicate that the dosage of estrogen supplement indicated by $\mu$ and $\rho$ and the frequency of estrogen supplement indicated by $1 / T$ play important roles in the treatment of osteoporosis patients. Even though the smaller value of $T$ yields the better result on the number of osteoclasts and osteoblasts, the side effects of estrogen administration are also needed to take into account. Therefore, the appropriate dosage and frequency of estrogen supplement that could control the number of bone-forming cells and bone-resorbing cells to lie within the desirable range might lead to an efficient treatment in osteoporosis patients.

\section{Data Availability}

The data/information supporting the formulation of the mathematical model in this paper are/is from previously reported studies and datasets, which have been cited in the paper. 


\section{Conflicts of Interest}

The authors declare that there are no conflicts of interest regarding the publication of this paper.

\section{Acknowledgments}

This work was supported by the Centre of Excellence in Mathematics, Thailand, Mahidol University, Thailand, and the Science Achievement Scholarship of Thailand (SAST).

\section{References}

[1] F. Cosman, S. J. de Beur, M. S. LeBoff et al., "Clinician's guide to prevention and treatment of osteoporosis," Osteoporosis International, vol. 25, no. 10, pp. 2359-2381, 2014.

[2] W. S. Group, "Prevention and management of osteoporosis," World Health Organization Technical Report Series, vol. 921, pp. 38-81, 2003.

[3] H. D. Nelson, C. D. Morris, D. F. Kraemer et al., "Osteoporosis in postmenopausal women: diagnosis and monitoring," Evidence Report/Technology Assessment (Summary), vol. 28, pp. 1-2, 2001.

[4] NIH Consensus Development Panel on Osteoporosis Prevention Diagnosis, and Therapy, "Osteoporosis prevention, diagnosis, and therapy," Journal of the American Medical Association: The Journal of the American Medical Association, vol. 285, no. 6, pp. 785-795, 2001.

[5] T. D. Rachner, S. Khosla, and L. C. Hofbauer, "Osteoporosis: now and the future," The Lancet, vol. 377, no. 9773, pp. 1276-1287, 2011.

[6] M.-X. Ji and Q. Yu, "Primary osteoporosis in postmenopausal women," Chronic diseases and translational medicine, vol. 1, no. 1, pp. 9-13, 2015.

[7] C. Cooper, G. Campion, and L. J. Melton 3rd, "Hip fractures in the elderly: a world-wide projection," Osteoporosis International, vol. 2, no. 6, pp. 285-289, 1992.

[8] J.-Y. Reginster and N. Burlet, "Osteoporosis: a still increasing prevalence,” Bone, vol. 38, no. 2, pp. 4-9, 2006.

[9] K. K. Limpaphayom, N. Taechakraichana, U. Jaisamrarn et al., "Prevalence of osteopenia and osteoporosis in Thai women," Menopause, vol. 8, no. 1, pp. 65-69, 2001.

[10] T. Sözen, L. Özışık, and N. Ç Başaran, “An overview and management of osteoporosis," European Journal of Rheumatology, vol. 4, no. 1, pp. 46-56, 2017.

[11] R. Florencio-Silva, G. Rodrigues da Silva Sasso, E. Sasso-Cerri, M. Jesus Simões, and P. Sérgio Cerri, "Biology of bone tissue: structure, function, and factors that influence bone cells," BioMed Research International, vol. 2015, Article ID 721746, 17 pages, 2015.

[12] United States Public Health Service, Bone Health and Osteoporosis: A Report of the Surgeon General, University Press of the Pacific, Honolulu, HI, USA, 2004.

[13] S. Jerez, S. Díaz-Infante, and B. Chen, "Fluctuating periodic solutions and moment boundedness of a stochastic model for the bone remodeling process," Mathematical Biosciences, vol. 299, pp. 153-164, 2018.

[14] Y. T. Isogai, T. Akatsu, T. Ishizuya et al., "Parathyroid hormone regulates osteoblast differentiation positively or negatively depending on differentiation stages," Journal of Bone and Mineral Research, vol. 11, pp. 1384-1393, 1996.

[15] J. E. Oniya, J. Bidwell, J. Herring, J. Hulman, and J. M. Hock, "In vivo, human parathyroid hormone fragment (hPTH 1-34) transiently stimulates immediate early response gene expression, but not proliferation, in trabecular bone cells of young rats," Bone, vol. 17, pp. 479-484, 1995.

[16] T. Ishizuya, S. Yokose, M. Hori et al., "Parathyroid hormone exerts disparate effects on osteoblast differentiation depending on exposure time in rat osteoblastic cells," Journal of Clinical Investigation, vol. 99, no. 12, pp. 2961-2970, 1997.

[17] M. Kroll, "Parathyroid hormone temporal effects on bone formation and resorption," Bulletin of Mathematical Biology, vol. 62, no. 1, pp. 163-188, 2000.

[18] D. W. Dempster, F. Cosman, M. Parisien, V. Shen, and R. Lindsay, "Anabolic actions of parathyroid hormone on bone," Endocrine Reviews, vol. 14, no. 6, pp. 690-709, 1993.

[19] M. E. Freeman, B. Kanyicska, A. Lerant, and G. Nagy, "Prolactin: structure, function, and regulation of secretion," Physiological Reviews, vol. 80, no. 4, pp. 1523-1631, 2000.

[20] P. Clément-Lacroix, C. Ormandy, L. Lepescheux et al., "Osteoblasts are a new target for prolactin: analysis of bone formation in prolactin receptor knockout mice," Endocrinology, vol. 140, no. 1, pp. 96-105, 1999.

[21] N. Charoenphandhu, K. Tudpor, K. Thongchote, W. Saengamnart, S. Puntheeranurak, and N. Krishnamra, "High-calcium diet modulates effects of long-term prolactin exposure on the cortical bone calcium content in ovariectomized rats," American Journal of Physiology-Endocrinology and Metabolism, vol. 292, no. 2, pp. E443-E452, 2007.

[22] H. M. Frost, "On the estrogen-bone relationship and postmenopausal bone loss: a new model," Journal of Bone and Mineral Research, vol. 14, no. 9, pp. 1473-1477, 1999.

[23] Y. Miyauchi, Y. Sato, T. Kobayashi et al., "HIF1 is required for osteoclast activation by estrogen deficiency in postmenopausal osteoporosis," Proceedings of the National Academy of Sciences, vol. 110, no. 41, pp. 16568-16573, 2013.

[24] D. Merlotti, L. Gennari, K. Stolakis, and R. Nuti, "Aromatase activity and bone loss in men," Journal of Osteoporosis, vol. 2011, Article ID 230671, 11 pages, 2011.

[25] J. A. Kants, "Treatment of osteoporosis in elderly women," The American Journal of Medicine, vol. 98, no. 2, pp. 60S-66S, 1995.

[26] B. L. Riggs and L. J. Melton, Osteoporosis: Etiology, Diagnosis, and Management, Lippincott Williams \& Wilkins, Philadelphia, PA, USA, 1995.

[27] M. E. Davis, N. M. Strandjord, and L. H. Lanzl, "Estrogens and the aging process," Jama, vol. 196, no. 3, pp. 219-224, 1966.

[28] C. Ciani, D. Sharma, S. B. Doty, and S. P. Fritton, "Ovariectomy enhances mechanical load-induced solute transport around osteocytes in rat cancellous bone," Bone, vol. 59, pp. 229-234, 2014.

[29] M. Chudtong, Y. Lenbury, and C. Rattanakul, "Stability, permanence and positive periodicity in a model of bone remodeling under impulsive PTH control," International Journal of Mathematical Models and Methods in Applied Sciences, vol. 5, pp. 77-86, 2011.

[30] C. Rattanakul, Y. Lenbury, N. Krishnamara, and D. J. Wollkind, "Modeling of bone formation and resorption mediated by parathyroid hormone: response to estrogen/PTH therapy," Biosystems, vol. 70, no. 1, pp. 55-72, 2003.

[31] C. Rattanakul and S. Rattanamongkonkul, "Effect of calcitonin on bone formation and resorption: mathematical modeling approach," System, vol. 3, no. 4, p. 2, 2011.

[32] W. Panitsupakamon and C. Rattanakul, "A delay-differential equation model of bone remodeling process: effects of estrogen supplements," International Journal of Mathematics and Computer Science, vol. 6, pp. 430-438, 2012. 
[33] I. Chaiya and C. Rattanakul, "Effects of prolactin on bone remodeling process with parathyroid hormone supplement: an impulsive mathematical model," Advances in Difference Equations, vol. 2017, p. 147, 2017.

[34] I. Chaiya and C. Rattanakul, "An impulsive mathematical model of bone formation and resorption: effects of parathyroid hormone, calcitonin and impulsive estrogen supplement," Advances in Difference Equations, vol. 2017, no. 1, p. 153, 2017.

[35] J. A. Albright and M. Sauders, The Scientific Basis of Orthopaedics, Appleton \& Lange, Norwalk, Connecticut, 1987.

[36] H. M. Goodman, Basic Medical Endocrinology, Elsevier Limited, Amsterdam, Netherlands, 2009.

[37] J. P. Raymond, R. Isaac, R. E. Merceron, and F. Wahbe, "Comparison between the plasma concentrations of prolactin and parathyroid hormone in normal subjects and in patients with hyperparathyroidism or hyperprolactinemia," Journal of Clinical Endocrinology \& Metabolism, vol. 55, no. 6, pp. 1222-1225, 1982.

[38] G. Weryha and J. Leclère, "Paracrine regulation of bone remodeling," Hormone Research, vol. 43, no. 1-3, pp. 69-75, 1995.

[39] P. Morley, J. Whitfield, and G. Willick, "Parathyroid hormone an anabolic treatment for osteoporosis," Current Pharmaceutical Design, vol. 7, no. 8, pp. 671-687, 2001.

[40] J. F. Whitfield, P. Morley, and G. E. Willick, The Parathyroid Hormone: An Unexpected Bone Builder for Treating Osteoporosis, Landes Bioscience Company, Austin, TX, USA, 1998.

[41] D. Seriwatanachai, N. Krishnamra, and J. P. T. M. van Leeuwen, "Evidence for direct effects of prolactin on human osteoblasts: inhibition of cell growth and mineralization," Journal of Cellular Biochemistry, vol. 107, no. 4, pp. 677-685, 2009.

[42] S. Karstrup, L. Hegedüs, and H. H. Holm, "Acute change in parathyroid function in primary hyperparathyroidism following ultrasonically guided ethanol injection into solitary parathyroid adenomas," Acta Endocrinologica, vol. 129, no. 5, pp. 377-380, 1993.

[43] K. Prank, H. Harms, M. Dammig, G. Brabant, F. Mitschke, and R. D. Hesch, "Is there low-dimensional chaos in pulsatile secretion of parathyroid hormone in normal human subjects?" American Journal of Physiology-Endocrinology and Metabolism, vol. 266, no. 4, pp. E653-E658, 1994.

[44] V. Lakshmikantham and P. S. Simeonov, Theory of Impulsive Differential Equations, Vol. 6, World Scientific, , Singapore, 1989.

[45] A. Lakmeche and O. Ario, "Birfurcation of non trivial periodic solutions of impulsive differential equations arising chemotherapeutic treatment," Dynamics of Continuous, Discrete and Impulsive Systems, vol. 7, pp. 265-287, 2000. 